\title{
Random walk methods for modeling hydrodynamic transport in porous and fractured media from pore to reservoir scale
}

\author{
Benoit Noetinger • Delphine Roubinet • \\ Jean-Raynald de Dreuzy • Anna Russian • \\ Philippe Gouze • Tanguy Le Borgne • \\ Marco Dentz • Frederick Delay
}

Received: date / Accepted: date

\begin{abstract}
Random walk (RW) or Continuous Time Random Walk (CTRW) are recurring Monte Carlo methods used to model convective and diffusive transport in complex heterogeneous media. Many applications can be found, including fluid mechanic, hydrology, and chemical reactors modeling. These methods are easy to implement, very versatile and flexible enough to become appealing for many applications because they generally overlook of deeply simplify the building of explicit complex meshes required by deterministic methods. RW and CTRW provide a good physical understanding of the interactions between the space scales of heterogeneities and the transport phenomena under consideration. In addition, they can result in efficient up-scaling methods, especially in context of flow and transport in fractured media. In the present study, we review the applications of RW or CTRW for several situations coping with various spatial scales, and different insights into up-scaling applications. RW and CTRW advantages and downsides are also discussed, thus providing a few avenues for further works and applications.
\end{abstract}

Keywords Random walk · Random Media - Fractured media · Diffusion . Dispersion · Up-scaling · Transfers · multiple porosity

B. Noetinger

IFPEN 1-4 avenue de Bois Preau, 92852, France

E-mail: benoit.noetinger@ifpen.fr

D. Roubinet

Applied and Environmental Geophysics Group, Institute of Earth Sciences, University of Lausanne, 1015 Lausanne, Switzerland

J.R. de Dreuzy

Géosciences Rennes

F. Delay

Laboratoire dHydrologie et de Géochimie de Strasbourg, Univ. Strasbourg/EOST, CNRS UMR 7517, 1 rue Blessig, 67000 Strasbourg, France 


\section{Introduction}

In the early 20 th century, thanks to seminal contributions of major scientists such as Einstein, Langevin, le Chatelier and Wiener among others, the Random walk (RW) approach introduced the first quantified depiction of brownian motion which resulted ii a fruitful microscopic picture of the diffusion mechanism [Scher et al., 2002a]. The conceptual simplicity of RW associated with its easiness of implementation were conducive to a fast dissemination of associated techniques and algorithms in many areas of science, from biology and colloidal science to modern finance, including statistical physics, chemical engineering, and geosciences. Regarding the latter topic, many applications can be found in the field of hydrology, oil and gas industry or subsurface repository of various wastes. As an illustration, RW techniques can also be used as a direct simulation technique of passive tracer or pollutant transport in aquifers[Kinzelbach, 1988, Kinzelbach and Uffink, 1991, Zimmermann et al., 2001, Hoteit et al., 2002b, Delay et al., 2005]. At the laboratory scale, the recent advances in the imaging techniques of rock textures provide high resolution pictures of the pore space [Nunes et al., 2015]. This very exhaustive and complex information needs for post-processing to infer effective transport properties in porous media such as permeability, porosity, and electrical conductivity, to mention a few. For example, electrical conductivity was first computed in using RW by [Kim and Torquato, 1992] and several authors ([Sahimi, 2011] and reference therein). At the same pore-to-sample scale, RW techniques were also used to interpret NMR data ([Néel et al., 2011, 2014, Guillon et al., 2013, 2014, Fleury et al., 2013]). At a larger scale, typically from $\mathrm{m}$ to $\mathrm{km}$, a major issue of various applications, especially those performing flow and transport simulations over subsurface reservoirs, is in the capability of accounting for the detailed natural variability of the host medium with the maximum accuracy (some $\mathrm{km}$ in the case of aquifers or oil \& gas reservoir). These applications range from a better understanding of the fate of pollutants in the subsurface, to improving oil and gas recovery. At the large scale, most calculations are still performed using Finite Volume, Finite Difference or Finite Element approaches requiring a high resolution and cumbersome meshing to represent the geometry of the modeled domain. However, because the size of an elementary mesh can be too large, discrete descriptions of the domain may also require the availability of effective transport equations at the scale of the mesh and accounting for eventual unresolved sub-grid scale effects. In most cases, it is assumed that transport is still ruled by the Advection Dispersion Equation (ADE) which is of questionable validity at the small scale [Matheron and De, 1980, Bouchaud and Georges, 1990, Berkowitz and Scher, 1998, Berkowitz et al., 2000, Néel et al., 2011, Scher et al., 2002a]. In addition, the parameters associated with flow and transport are usually poorly known a priori and need to be assessed by facing model outputs with available data. RW techniques are good candidates for both identifying sub-grid effects and parameters ([Berkowitz and Balberg, 1993, Néel et al., 2011]). In particular, obtaining valuable descriptions of transport in low permeability media concealing widespread heterogeneities involving stagnant zones, adsorption and chemical reactions properties is of major interest. These highly disordered media can exhibit very rich anomalous transport properties preventing any classical description relying upon standard Darcy's law and ADE. RW methods can for instance represent "fractional derivative" transport equation accounting for memory effects over wide time ranges. [Matheron and De, 
1980, O'Shaughnessy and I. Procaccia, 1985, Charlaix et al., 1987, Barker, 1988, Chang et al., 1990, Bouchaud and Georges, 1990, Metzler et al., 1994, Noetinger and Gautier, 1998, Metzler and J. Klafter, 2000]. In the extreme case of fractured media, Continuous Time Random Walk (CTRW) or Time Domain Random Walk (TDRW) algorithms techniques were succesfully applied to determine transfer functions useful for implementing so called dual porosity modelsNoetinger and Estebenet [2000], Noetinger et al. [2001b,a].

RW algorithms are very well suited to capture the motion and the spreading of a diffusive set of particles representing a tracer plume in an advecting flow field. The rather subtle interplay between molecular diffusion and the heterogeneous velocity field can be simulated directly, providing a clear physical understanding of the relevant characteristic times and scales prevailing during transport. Analytical results of the Taylor and Aris [Taylor, 1954, Aris, 1956] dispersion can be retrieved. In turn, these results can be used to estimate dispersivity in more complex geometries. In the case of spatially varying diffusion coefficients, including discontinuous cases, the RW may experience some difficulties. To avoid spurious accumulation of particles in low diffusivity zones, some "reflection rules" have to be imposed at the locations of discontinuities. [Kinzelbach, 1988, Kinzelbach and Uffink, 1991, Hoteit et al., 2002b]. In addition, the algorithm can become inefficient in low diffusivity zones because the spatial time step needed for an accurate resolution of transport can become very small. Both issues can be corrected using suitable time step increments that avoid expansive and unnecessary iterations in low velocity and low diffusion zones.

Anomalous dispersion effects mainly due the persistence of large scale correlations in a flow field can thus be simulated using CTRW with suitable kernels [Berkowitz and Scher, 1998, Berkowitz et al., 2000, Néel et al., 2011, Scher et al., 2002a,b]. . Regarding their numerical part, CTRW methods can be implemented using structured or unstructured meshes, which allows to tackle the problem of diffusion in very complex media, including fracture networks [Noetinger and Estebenet, 2000, Noetinger et al., 2001b,a, Roubinet et al., 2010, 2013]. Adsorption phenomena, as well as chemical reactions can be accounted for. By nature, RW and CTRW are very well suited for highly parallel computing architectures which can be useful to perform calculations over complex domains or when moving multiple reacting species in solution.

RW and CTRW methods provide interesting insights into transport mechanisms that can enlighten us about our understanding of mixing processes in disordered flows. In the case of fractured media, a careful interpretation of the output of CTRW simulations give efficient and direct tools to parameterize multiple porosity large scale models by way of a complete determination of transient transfer functions [Noetinger and Estebenet, 2000, Noetinger et al., 2001b,a, Roubinet et al., 2010, 2013]. These functions can thus help to set up multiple rate transfer models [Haggerty and Gorelick, 1995]or Multiple Interacting Continua (MINC) models [Narasimhan and Pruess, 1988]. Characteristic exponents associated with anomalous diffusion/dispersion processes can be estimated using RW methods [Arcangelis et al., 1986, Berkowitz and Scher, 1997, Carthy, 1993b, Koplik et al., 1988, Bouchaud and Georges, 1990], as well as the REV or mixing lengths, even if large scale practical simulations still remain the playing field of conventional meshed models. 
The goal of the present paper is to review the state of the art regarding applications of RW, TDRW or CTRW to the up scaling of flows and mass transfers in heterogeneous and fractured media.

The contribution is organized as follows. We first review the underlying theory of RW and TDRW algorithms for solving an advection diffusion equation. Next, numerical issues are addressed, from fully coupled advection dispersion diffusion equations to purely diffusive issues including heterogeneous, fractured media and the double porosity large scale description. Finally applications from pore to field scale, including radial flows to a well.

\section{Theoretical Background for Random Walk Methods}

This section summarizes the basis on which random walk methods to describe solute transport in heterogeneous media are built on. We start with classical or discrete time random walks, then briefly review its generalization to continuous time, the continous time random walk (CTRW) framework. Based on the CTRW approach, we report the bases of the time domain random walk method (TDRW). Finally, we review the use of the CTRW as an average transport framwork for transport in heterogeneous media.

\subsection{Classical (Discrete-Time) Random Walks}

Classical random walk particle tracking is based on the equivalence between the Fokker-Planck equation and the equation of motion of solute particles, which move due to advective and diffusive-dispersive mass transfer. A general Fokker-Planck equation for the scalar quantity $P(\mathbf{x}, t)$ can be written as [Risken, 1996]

$$
\frac{\partial P(\mathbf{x}, t)}{\partial t}+\nabla \cdot[\mathbf{v}(\mathbf{x}) P(\mathbf{x}, t)]+\nabla \otimes \nabla:[\mathbf{B}(\mathbf{x}) P(\mathbf{x}, t)]=0,
$$

where $\otimes$ denotes the outer and : the inner tensor product. The drift and dispersion tensor are denoted by $\mathbf{v}(\mathbf{x})$ and $\mathbf{B}(\mathbf{x})$. As shown in Appendix A, this Fokker-Planck equation is exactly equivalent to the Langevin equation

$$
\frac{d \mathbf{x}(t)}{d t}=\mathbf{v}[\mathbf{x}(t)]+\sqrt{2 \mathbf{B}[\mathbf{x}(t)]} \cdot \zeta(t)
$$

where $\sqrt{\mathbf{B}(\mathbf{x})}$ is the square-root of the tensor $\mathbf{B}(\mathbf{x}), \boldsymbol{\zeta}(t)$ denotes a Gaussian white noise characterized by zero mean $\langle\boldsymbol{\zeta}(t)\rangle=\mathbf{0}$ and variance $\left\langle\zeta_{i}(t) \zeta_{j}\left(t^{\prime}\right)\right\rangle=\delta_{i j} \delta\left(t-t^{\prime}\right)$. The angular brackets denote the noise average over all particles. The particle distribution $P(\mathbf{x}, t)$ can be written in terms of the particle trajectories $\mathbf{x}(t)$ as

$$
P(\mathbf{x}, t)=\langle\delta[\mathbf{x}-\mathbf{x}(t)]\rangle .
$$

We call this modeling approach also a discrete time random walk because particle positions are incremented in constant time intervals as

$$
\mathbf{x}(t+d t)=\mathbf{x}(t)+\mathbf{v}[\mathbf{x}(t)] d t+\sqrt{2 \mathbf{B}[\mathbf{x}(t)]} \cdot \mathbf{w}(t), \quad \mathbf{w}(t)=\int_{t}^{t+d t} d t^{\prime} \boldsymbol{\zeta}\left(t^{\prime}\right) .
$$


Note that we use here the Ito interpretation of the stochastic integral [Risken, 1996]. The random increment $\mathbf{w}(t)$ has zero mean and variance $\left\langle w_{i}(t) w_{j}(t)\right\rangle=$ $\delta_{i j} d t$.

Solute or heat transport as well as hydraulic head propagation in a heterogeneous porous medium, however, is in general not described by a Fokker-Planck equation of the form (1), but the advection-dispersion equation (ADE) for the scalar $c(\mathbf{x}, t)$

$$
\phi(\mathbf{x}) \frac{\partial c(\mathbf{x}, t)}{\partial t}+\nabla \cdot[\mathbf{u}(\mathbf{x}) c(\mathbf{x}, t)]-\nabla \cdot[\mathbf{D}(\mathbf{x}) \nabla c(\mathbf{x}, t)]=0
$$

For solute transport $c(\mathbf{x}, t)$ denotes concentration, $\phi(\mathbf{x})$ porosity and $\mathbf{D}(\mathbf{x})$ is the hydrodynamic dispersion tensor. For hydraulic head propagation, we set the flow velocity $\mathbf{u}(\mathbf{x})=\mathbf{0}, \phi(\mathbf{x})$ denotes specific storage and $\mathbf{D}(\mathbf{x})$ denotes the hydraulic conductivity tensor. It is important to note that $c(\mathbf{x}, t)$ in $(5)$ is not a conserved quantity because (5) is not mass conservative. Thus, we define the conserved

$$
P(\mathbf{x}, t)=\phi(\mathbf{x}) c(\mathbf{x}, t) .
$$

The latter satisfies the equation

$$
\frac{\partial P(\mathbf{x}, t)}{\partial t}+\nabla \cdot\left[\frac{\mathbf{u}(\mathbf{x})}{\phi(\mathbf{x})} P(\mathbf{x}, t)\right]-\nabla \cdot\left[\mathbf{D}(\mathbf{x}) \nabla \frac{P(\mathbf{x}, t)}{\phi(\mathbf{x})}\right]=0 .
$$

We can reformulate this equation in the form of the Fokker-Planck equation (1) as

$$
\frac{\partial P(\mathbf{x}, t)}{\partial t}+\nabla \cdot\left[\frac{\mathbf{u}(\mathbf{x})}{\phi(\mathbf{x})}+\frac{\nabla \cdot \mathbf{D}(\mathbf{x})}{\phi(\mathbf{x})}\right] P(\mathbf{x}, t)-\nabla \otimes \nabla:\left[\frac{\mathbf{D}(\mathbf{x})}{\phi(\mathbf{x})} P(\mathbf{x}, t)\right]=0 .
$$

Through the equivalence between (1) and (2), we find the Langevin equation that is equivalent to (5) as [Kinzelbach, 1988]

$$
\frac{d \mathbf{x}(t)}{d t}=\frac{\mathbf{u}[\mathbf{x}(t)]}{\phi[\mathbf{x}(t)]}+\frac{\nabla \cdot \mathbf{D}[\mathbf{x}(t)]}{\phi[\mathbf{x}(t)]}+\sqrt{\frac{2 \mathbf{D}[\mathbf{x}(t)]}{\phi[\mathbf{x}(t)]}} \cdot \zeta(t) .
$$

The solute concentration $c(\mathbf{x}, t)$ is given in terms of the particle trajectories through (3) and (6) as

$$
c(\mathbf{x}, t)=\frac{\langle\delta[\mathbf{x}-\mathbf{x}(t)]\rangle}{\phi(\mathbf{x})}
$$

\subsection{Continuous Time Random Walks}

We present here a generalization of the previous discrete time random walk picture to continuous time [Berkowitz et al., 2002, 2006]. The particle position and time are now incremented at each random walk step as

$$
\mathbf{x}_{n+1}=\mathbf{x}_{n}+\mathbf{A}\left(\mathbf{x}_{n}\right), \quad t_{n+1}=t_{n}+\tau\left(\mathbf{x}_{n}\right) .
$$


The random space and time increments $\mathbf{A}\left(\mathbf{x}_{n}\right)$ and $\tau\left(\mathbf{x}_{n}\right)$ can in general depend on the particle position and they can be coupled [Scher and Lax, 1973a], such that they are characterized by the joined probability density function (PDF) $\psi(a, t ; \mathbf{x})$. Notice that both space and time are continuous variables. The particle density $P(\mathrm{x}, t)$ can be described by the following system of equations [Berkowitz et al., 2002, Srinivasan et al., 2010]

$$
\begin{aligned}
& P(\mathbf{x}, t)=\int_{0}^{t} d t^{\prime} R\left(\mathbf{x}, t^{\prime}\right) \int_{t-t^{\prime}}^{\infty} d t^{\prime \prime} \psi\left(t^{\prime \prime} ; \mathbf{x}\right) \\
& R(\mathbf{x}, t)=P(\mathbf{x}, t) \delta(t)+\int_{d} d \mathbf{a} \int_{0}^{t} d t^{\prime} \psi\left(\mathbf{a}, t^{\prime} ; \mathbf{x}-\mathbf{a}\right) R\left(\mathbf{x}-\mathbf{a}, t-t^{\prime}\right) .
\end{aligned}
$$

The first equation states that the probability of a particle to be in $[\mathbf{x}, \mathbf{x}+d \mathbf{x}]$ at time $t$ is equal to the probability $R\left(\mathbf{x}, t^{\prime}\right)$ that the particle is in $[\mathbf{x}, \mathbf{x}+d \mathbf{x}]$ in $\left[t^{\prime}, t^{\prime}+d t^{\prime}\right]$ and remains there for the duration of $t-t^{\prime}$. The marginal PDF of transition times is given by

$$
\psi(t ; \mathbf{x})=\int d \mathbf{a} \psi(\mathbf{a}, t ; \mathbf{x})
$$

The second equation can be seen as a Chapman Kolmogorov equation for the probability density $R(\mathbf{x}, t)$. The linear system (12) can be solved for the Laplace transform of the particle density $P(\mathbf{x}, t)$, which gives after some algebra

$$
\begin{aligned}
\lambda P(\mathbf{x}, \lambda) & =P(\mathbf{x}, 0)+\int d \mathbf{a} \frac{\lambda \psi(\mathbf{a}, \lambda ; \mathbf{x}-\mathbf{a})}{1-\psi(\lambda ; \mathbf{x}-\mathbf{a})} P(\mathbf{x}-\mathbf{a}, \lambda) \\
& \int d \mathbf{a} \frac{\lambda \psi(\mathbf{a}, \lambda ; \mathbf{x})}{1-\psi(\lambda ; \mathbf{x})} P(\mathbf{x}, \lambda)
\end{aligned}
$$

Inverse Laplace transform gives the generalized Master equation [Kenkre et al., 1973]

$$
\begin{aligned}
\frac{d P(\mathbf{x}, t)}{d t} & =\int_{0}^{t} d t^{\prime} \int d \mathbf{a} M(\mathbf{a}, t ; \mathbf{x}-\mathbf{a}) P(\mathbf{x}-\mathbf{a}, t) \\
& -\int_{0}^{t} d t^{\prime} \int d \mathbf{a} M(\mathbf{a}, t ; \mathbf{x}-\mathbf{a}) P(\mathbf{x}, t),
\end{aligned}
$$

where we defined the memory function

$$
M(\mathbf{a}, \lambda ; \mathbf{x})=\frac{\lambda \psi(\mathbf{a}, \lambda ; \mathbf{x})}{1-\psi(\lambda ; \mathbf{x})}
$$

Under the assumption that $M(\mathbf{a}, t ; \mathbf{x}-\mathbf{a})$ is sharply peaked about $\mathbf{0}$ the spatial convolution in (15) may be localized trough a Taylor expansion of the integrand 
about $\mathrm{x}$ [Berkowitz et al., 2002] such that

$$
\begin{aligned}
& \frac{\partial P(\mathbf{x}, t)}{\partial t}+\int_{0}^{t} d t^{\prime} \nabla \cdot\left[\boldsymbol{\nu}\left(\mathbf{x}, t-t^{\prime}\right) P\left(\mathbf{x}, t^{\prime}\right)\right] \\
& -\int_{0}^{t} d t^{\prime} \nabla \otimes \nabla \cdot\left[\boldsymbol{\beta}\left(\mathbf{x}, t-t^{\prime}\right) P\left(\mathbf{x}, t^{\prime}\right)\right]+\cdots=0,
\end{aligned}
$$

where the dots represent higher order contributions in the moments of $M(\mathbf{a}, t ; \mathbf{x})$. We defined the velocity and dispersion kernels $\boldsymbol{\nu}(\mathbf{x}, t)$ and $\boldsymbol{\beta}(\mathbf{x}, t)$ as

$$
\nu(\mathbf{x}, t)=\int d \mathbf{a} \mathbf{a} M(\mathbf{a}, t ; \mathbf{x}), \quad \boldsymbol{\beta}(\mathbf{x}, t)=\int d \mathbf{a} \mathbf{a} \otimes \mathbf{a} M(\mathbf{a}, t ; \mathbf{x})
$$

\subsection{Time-Domain Random Walks}

Time domain random walk (TDRW) methods have been used as efficient alternatives to discrete time random walks for the solution of solute transport and flow problems in heterogeneous media as described by (5). Complementary to the classical discrete time random walk approach, the TDRW fixes the spatial particle transition length and asks for the time that is needed to traverse this distance. Evidently, this approach is in the spirit of the CTRW discussed in the previous section. In order to elucidate this relation, let us consider the ADE (5) discretized in space. Using a finite difference or finite volume discretization, it can be written as as [Delay et al., 2002, Dentz et al., 2012, Russian et al., 2016]

$$
\phi_{i} \frac{d c_{i}(t)}{d t}=\sum_{[i j]} b_{i j} c_{j}(t)-\sum_{[i j]} b_{j i} c_{i}(t),
$$

where $c_{i}(t)$ is the concentration at voxel $i$. The notation $\sum_{[i j]}$ means summation over the nearest neighbors of voxel $i$. The coefficients $b_{i j}$ are given in terms of the flow velocity and dispersion coefficients as [Gjetvaj et al., 2015, Russian et al., 2016]

$$
b_{i j}=\frac{\hat{D}_{i j}}{\xi^{2}}+\frac{\left|u_{i j}\right|}{2 \xi}\left(\frac{u_{i j}}{\left|u_{i j}\right|}+1\right),
$$

with the discretization length $\xi$. We assume for simplicity that the discretization is uniform and all grid cells, or voxels, have the same volume $V$ and surface area $S$. The velocity component $u_{i j}$ of $\mathbf{u}_{j}$ is the flow velocity at voxel $j$ in direction of voxel $i$, that is $u_{i j}=\mathbf{u}_{j} \cdot \mathbf{e}_{i j}$, where the vector $\mathbf{e}_{i j}$ points from voxel $j$ to voxel $i$. Thus, $u_{i j}>0$ implies that voxel $i$ is downstream from voxel $j$, and vice versa. The dispersion coefficient $\hat{D}_{i j}$ measures the dispersive mass flux between voxels $i$ and $j$. It is typically given by a suitable average of the dispersion coefficient in neighboring voxels [Noetinger and Estebenet, 2000, Dentz et al., 2012]. 
In order to see the equivalence with the CTRW approach, we proceed as in Dentz et al. [2012] and write the generalized Master equation (15) in discrete space,

$$
\frac{d P_{i}(t)}{d t}=\int_{0}^{t} d t^{\prime} \sum_{[i j]}\left[M_{i j}\left(t-t^{\prime}\right) P_{j}\left(t^{\prime}\right)-M_{j i}\left(t-t^{\prime}\right) P_{i}\left(t^{\prime}\right)\right]
$$

where we assume that there are interactions only between nearest neighbors. Furthermore, we set $P\left(\mathbf{x}_{i}, t\right)=P_{i}(t)$ and $M\left(\mathbf{x}_{i}-\mathbf{x}_{j}, t ; \mathbf{x}_{j}\right)=M_{i j}(t)$. We assume now that the transition length and time PDF can be decoupled into $\psi_{i j}(t)=w_{i j} \psi_{j}(t)$ with $w_{i j}$ the probability to make a transition from $j$ to $i$. We furthermore specify the exponential transition time PDF [Scher and Lax, 1973a]

$$
\psi_{j}(t)=\exp \left(t / \tau_{j}\right) / \tau_{j} .
$$

Thus, Laplace transform of the memory function $M_{i j}(t)$ simplifies to

$$
M_{i j}(t)=\frac{w_{i j}}{\tau_{j}}
$$

The generalized Master equation then reads as

$$
\frac{d P_{i}(t)}{d t}=\sum_{[i j]} \frac{w_{i j}}{\tau_{j}} P_{j}(t)-\sum_{[i j]} \frac{w_{j i}}{\tau_{i}} P_{i}(t)
$$

Identifying now $P_{i}(t)=\phi_{i} c_{i}(t)$ and $b_{i j}=w_{i j} \phi_{j} / \tau_{j}$, (19) and (24) are identical. This means the discretized ADE (19) can be solved through a TDRW, whose transition probability in space and transition time are given by

$$
w_{i j}=\frac{b_{i j} \tau_{j}}{\phi_{j}}, \quad \tau_{i}=\frac{\phi_{i}}{\sum_{[i j]} b_{j i}} .
$$

2.4 The Continuous Time Random Walk as an Average Transport Approach

The CTRW approach has been used as an upscaling framework to describe average transport in heterogeneous media [Klafter and Silbey, 1980, Berkowitz et al., 2006]. The pioneering work of Scher and Lax [1973a] and Scher and Lax [1973b] models the transport of charge carries in impure semiconductors with a CTRW whose PDF of waiting times reflects trapping in the potential wells created by charged impurities. This approach allowed for the explanation and prediction of observed charge currents, or in other words, arrival time distributions, which show heavy tails characteristic for anomalous transport. Since then, the CTRW has been used as an approach to model the history-dependent average dynamics in wide range of fluctuating and disordered systems inlcuding particle transport in pore-scale and Darcy scale heterogeneous media [Berkowitz and Scher, 1998, 1997, Hatano and Hatano, 1998, Berkowitz et al., 2000, Cortis and Berkowitz, 2004, Le Borgne et al., 2008b, De Anna et al., 2013, Kang et al., 2014, Holzner et al., 2015], the dispersion of light in heterogeneous optical media [Barthelemy et al., 2008], the 
description of financial distributions, animal motion, and many more [Klafter and Sokolov, 2005].

The CTRW as an average transport or upscaling approach for heterogeneous media, maps the spatial distributions of the fluctuating medium properties into the (joint) distribution of transition length and times $\psi(\mathbf{a}, t)$. Notice that in general the joint PDF depends on the spatial position, as in Sections 2.2 and 2.3. This spatial dependence is here homogenized by the ensemble average as outlined in Scher and Lax [1973a] and Scher and Lax [1973b]. In order to illustrate this approach, we adopt the method of Appendix B in Scher and Lax [1973a] for pure diffusion in a medium characterized by spatially distributed particle traps [see also, Bouchaud and Georges, 1990, Dentz et al., 2016a]. Our starting point is equation (19) for a medium that is characterized by constant and isotropic dispersion properties, but a spatially variable porosity, or retardation coefficient $\phi_{i}$, which quantifies the strength of the particle trap. Under these conditions, the coefficients $b_{i j}$ in (20) simplify to

$$
b=\frac{D}{\xi^{2}}
$$

Thus, the CTRW for this transport system is characterized by the joint transition PDF

$$
\psi_{i j}(t)=\frac{1}{2 d} \psi_{j}(t), \quad \psi_{j}(t)=\exp \left(-t / \tau_{j}\right) / \tau_{j}, \quad \tau_{j}=\phi_{j} \tau_{D}
$$

where we defined $\tau_{D}=\xi^{2} / D ; d$ is the dimension of space. Thus, equations (12) read for this CTRW

$$
\begin{aligned}
& P_{i}(t)=\int_{0}^{t} d t^{\prime} R_{i}\left(t^{\prime}\right) \int_{t-t^{\prime}}^{\infty} d t^{\prime \prime} \psi_{i}\left(t^{\prime \prime}\right) \\
& R_{i}(t)=P_{0}(t) \delta(t)+\sum_{[i j]} \int_{0}^{t} d t^{\prime} \frac{1}{2 d} \psi_{j}\left(t-t^{\prime}\right) R_{j}\left(t^{\prime}\right) .
\end{aligned}
$$

The average transport behavior can now be obtained by an ensemble average over the disorder, this means here over the distribution over the $\phi_{i}$. To this end, we assume that the $\phi_{i}$ at different sites $i$ and $j$ are uncorrelated. Now, we recall that the $R_{i}(t)$ represents the probability per time that a particle has just arrived at site $i$. Thus, it integrates information of all the other sites visited before. We further recall now that the average number of new sites visited for an isotropic lattice random walk in $d>3$ spatial dimensions increases with the number of random walk steps [Bouchaud and Georges, 1990]. This means that $R_{i}(t)$ and $\psi_{i}(t)$ can be assumed to be statistically independent because it is not very likely that a particle returns to the same site. Thus, for $d=3$ dimensions we can perform the ensemble average through an integration of $(28)$ over the distribution $p_{\phi}(\phi)$ of point values 
of $\phi_{i}$, such that we obtain

$$
\begin{aligned}
& \bar{P}_{i}(t)=\int_{0}^{t} d t^{\prime} \bar{R}_{i}\left(t^{\prime}\right) \int_{t-t^{\prime}}^{\infty} d t^{\prime \prime} \psi\left(t^{\prime \prime}\right) \\
& \bar{R}_{i}(t)=P_{0}(t) \delta(t)+\sum_{[i j]} \int_{0}^{t} d t^{\prime} \frac{1}{2 d} \psi\left(t-t^{\prime}\right) \bar{R}_{j}\left(t^{\prime}\right),
\end{aligned}
$$

where the PDF of transition times integrates the disorder distributions as

$$
\psi(t)=\int_{0}^{\infty} d \phi p_{\phi}(\phi) \frac{\exp \left(-\frac{t}{\phi \tau_{D}}\right)}{\phi \tau_{D}} .
$$

Recall that the conditions for such an average transport description is that the disorder experienced by the particle at subsequent steps needs to be independent.

In this spirit, CTRW has been applied to advective and dispersive transport in heterogeneous media. For advection-dominated transport, the transition time $\tau$ is related to the advection time over a characteristic length $\ell$, which is of the order of the correlation distance of the particle velocity along a streamline, such that

$$
\tau=\frac{\ell}{v}
$$

where $v$ is the particle velocity. The spatial correlation properties of Lagrangian particle velocities along streamlines, and the representation of transport by CTRW have been studied for pore and Darcy scale transport [Le Borgne et al., 2008a,b, Kang et al., 2011, De Anna et al., 2013, Kang et al., 2014]. The relation between the spatial distribution of hydraulic conductivity and transition times has been studied in Edery et al. [2014]. The CTRW as an average transport approach has been used for the interpretation and prediction of a wide range of anomalous and non-Fickian behaviors observed in geological media [Berkowitz et al., 2006]. In the following, we briefly summarize the behaviors of breathrough curves and spatial concentration moments that are obtained in the CTRW framework for a transition time PDF that decays algebraically as $\psi(t) \propto t^{-1-\beta}$ with $0<\beta<2$.

\subsubsection{Breakthrough Curves}

Hydrodynamic transport in natural formations is frequently characterized by breakthrough curves, this means first passage time PDFs, or solute fluxes. Both are essentially the same in the case of advectively dominated transport. The first passage time $t\left(x_{1}\right)$ at a control plane located at $x_{1}$ can be formulated in terms of particle trajectories as

$$
t\left(x_{1}\right)=\inf \left[t \mid x(t) \geq x_{1}\right] .
$$

The PDF of first passage times then can be written as

$$
f\left(t, x_{1}\right)=\left\langle\delta\left[t-t\left(x_{1}\right)\right]\right\rangle .
$$


For an instantaneous particle injection into an infinite or semi-infinite domain, it has been found [Berkowitz et al., 2006] that $f\left(t, x_{1}\right)$ scales as the transition time PDF,

$$
f\left(t, x_{1}\right) \propto t^{-1-\beta}
$$

\subsubsection{Spatial Concentration Moments}

Other observables that characterize the global plume behavior are the first and second centered spatial moments of the solute distribution. The first $m_{i}^{(1)}$ and second $m_{i j}^{(2)}$ spatial moments of $P(\mathbf{x}, t)$ are defined as

$$
m_{i}^{(1)}(t) \equiv \int d^{d} x x_{i} P(\mathbf{x}, t) \quad m_{i j}^{(2)}(t) \equiv \int d^{d} x x_{i} x_{j} P(\mathbf{x}, t)
$$

In terms of the particle trajectories, they are given by $m_{i}^{(1)}(t)=\left\langle x_{i}(t)\right\rangle$ and $m_{i j}^{(2)}(t)=\left\langle x_{i}(t) x_{j}(t)\right\rangle$. The second centered moments are a measure for the global plume width, and are defined by

$$
\kappa_{i j}(t)=m_{i j}^{(2)}(t)-m_{i}^{(1)}(t) m_{j}^{(1)}(t) .
$$

For the time behavior of the spatial moments, we distinguish the $\beta$-ranges between 0 and 1 and 1 and 2. For $0<\beta<1$ it has been found that [Berkowitz et al., 2006]

$$
m_{1}(t) \propto t^{\beta}, \quad \kappa_{11}(t) \propto t^{2 \beta}, \quad \kappa_{22}(t) \propto t^{\beta} .
$$

In the range $1<\beta<2$ the moments behave as

$$
m_{1}(t) \propto t, \quad \kappa_{11}(t) \propto t^{3-\beta}, \quad \kappa_{22}(t) \propto t .
$$

These behaviors are valid in an asymptotic sense if there are time regimes, for which the transition time PDF shows the power-law behavior $\psi(t) \propto t^{-1-\beta}$ for $0<\beta<2$. For $\beta>2$ the breakthrough and dispersion behavior is asymptotically Fickian. Note also that one would expect that there exists a cut-off scale, maybe linked to the largest velocity scale, after which the transition time PDF decreases faster than a power-law. Such situations and the transitions form anomalous to normal transport behaviors have been investigated in [Dentz et al., 2004].

\section{Numerical Random Walk Particle Tracking Methods}

\subsection{Classical Random Walks}

The simplest random particle tracking algorithm to solve the ADE (5) is obtained by time discretization of the Langevin equation (9) using the Ito convention of the stochastic integral,

$$
\mathbf{x}(t+\Delta t)=\mathbf{x}(t)+\frac{\mathbf{u}[\mathbf{x}(t)]}{\phi[\mathbf{x}(t)]} \Delta t+\frac{\nabla \cdot \mathbf{D}[\mathbf{x}(t)]}{\phi[\mathbf{x}(t)]} \Delta t+\sqrt{\frac{2 \mathbf{D}[\mathbf{x}(t)] \Delta t}{\phi[\mathbf{x}(t)]}} \cdot \mathbf{Z}(t),
$$


which yields the Euler scheme. In the preceding equation, the time step is supposed to be discretized in increments $d t$. In the basic version, time steps are assumed to be constant. The $\mathbf{Z}(t)$ are independent identically distributed Gaussian random vectors with zero mean, $\langle\mathbf{Z}(t)\rangle=\mathbf{0}$ and covariance $\left\langle Z_{i}(t) Z_{j}(t)\right\rangle=\delta_{i j}$. In practice, the random noise $\mathbf{Z}(t)$ may be replaced by any random vector with mean zero and variance 1 . In the heterogeneous case, including media characterized by discontinuous properties, the above formulation has several drawbacks. Intuitively, one can anticipate spurious phenomena because of the Dirac delta function that appears in the evaluation of the gradient of discontinuous diffusivity. In the present form of RW, to account for these diffusivity discontinuities requires the implementation of quite complex "reflection" rules of the RW at the interface between two media Kinzelbach and Uffink [1991], Kinzelbach [1988], Zimmermann et al. [2001], Hoteit et al. [2002b]. A second drawback is that in very low diffusion coefficient zones, the basic algorithm leads to inefficient small spatial displacements. The particles remain trapped in low diffusion zones, increasing the effective computational cost which is proportional to the number of time iterations.

These drawbacks can be avoided by using alternative versions of the basic RW algorithm. The main idea is using fixed spatial meshes instead of even time steps. This leads to model diffusion over a fixed lattice or graph that can be built with standard meshing tools. For its part, the residence time on a given site becomes a random variable, with the meaning that the resulting algorithm belongs to a wider class of algorithms, namely the Time Domain Random Walk (TDRW). These TDRW algorithms are also particularly well suited to implementations on highly parallel computing resources. An additional interest is that the resulting formulation is quite close to the classical solutions as that provided by standard numerical analysis.

\subsection{Fully coupled advection-dispersion-diffusion equation}

Introducing advective and dispersive terms in the same equation raises specific numerical issues mostly linked to discontinuities in the dispersion gradient and to the coupling between the first-order advective term and the second-order diffusivedispersive term. Nonetheless, RW methods remain widely used because of their good numerical properties, irrespective the rate of advection to diffusion-dispersion and also because they are easily implemented. Time domain variants have drastically improved the performances of RW methods, especially in heterogeneous media. At the local scale, hydrodynamic dispersion is generally modeled as a Fickian diffusion with the diffusion-dispersion coefficient $\mathrm{D}(\mathbf{x})$ of equation (1) expressed as a tensor of components Bear [1973]:

$$
D_{i j}=\left(\alpha_{T}|\mathbf{v}|+d\right) \delta_{i j}+\left(\alpha_{L}-\alpha_{T}\right) \frac{v_{i} v_{j}}{|\mathbf{v}|}
$$

where d is the diffusion coefficient, $D_{L}$ and $D_{T}$ are the longitudinal and transverse dispersivities, $v_{i}$ is the velocity in direction $x_{i},|\mathbf{v}|$ is the norm of the velocity, and $\delta_{i j}$ is the Kronecker symbol. Along the principal directions of dispersion, this 
expression simplifies into

$$
D=\left[\begin{array}{ccc}
d+\alpha_{L}|\mathbf{v}| & 0 & 0 \\
0 & d+\alpha_{T}|\mathbf{v}| & 0 \\
0 & 0 & d+\alpha_{T}|\mathbf{v}|
\end{array}\right]
$$

where the first coordinate is taken along the direction given by the velocity and the two other directions are in the plane normal to the velocity

Therefore, both the advective and dispersive terms require the determination of the velocity $\mathrm{v}$ at any location the modeled domain, while Eulerian methods only require the knowledge of velocities at the scale of the mesh cell. When the velocity field has been obtained numerically from the discretization of the flow equation over a calculation grid, the velocity is expressed within each mesh cell with appropriate methods. If the numerical scheme is based on flow conservative Finite Element methods (e.g., Mixed Finite Element, Mixed Hybrid Finite Element) [Brezzi and Fortin, 1991, Hoteit et al., 2002a, Mosé et al., 1994, Pichot et al., 2010, Roberts and $\mathrm{m}$. Thomas, 1991], velocity is directly derived from a combination of the finiteelement basis functions [Hoteit et al., 2002b]. With more standard Finite Element approaches, conservative velocity scheme can still be derived using flux-conserving sub-cell subdivisions [Cordes and Kinzelbach, 1992, 1996]. For Finite Difference and Finite Volume schemes, velocities are most commonly calculated within mesh cells with multi-linear interpolations (linear along the different directions of the meshing) [Pollock, 1988]. In fact, only the linear interpolation scheme is fluxconservative. In the specific cases handling regular grids, the linear interpolation scheme can also be derived from the equivalence between Finite Volume methods and Mixed Hybrid Finite Element schemes [Chavent and Roberts, 1991]. While mass-conservative transport schemes require flux-conservative schemes, they do not impose any condition to the transverse velocity across mesh faces that appear in general to be discontinuous. Associated with multi-linear interpolation schemes, shear deformation accumulates in the edges of the mesh cell and is zero outside. This is a strong limitation when studying solute mixing mechanisms with geometrical and process-based analysis Borgne and T. [2015]. Alternative quadratic velocity interpolation schemes have been proposed to ensure non-slip boundary conditions at impervious limits Nunes et al. [2015]. Mass conservation conditions are no longer fulfilled locally but they are globally, over cell facets. Such type of interpolation scheme is appropriate in the presence of numerous no-flow boundary conditions (e.g. at the pore scale).

While classical Eulerian schemes only need the expression of the dispersion D Zheng and Bennett [2002], random walk methods also require the dispersion gradient (equation (2)), and thus the velocity gradient. This is an important specificity of RW methods that conditions the choice of both the numerical schemes and implementation methods. The main difficulty stems from the discontinuity of the velocity gradient and hence of the dispersion gradient across cells. We first underline that the effect of the discontinuity at the cell facet is not systematically critical and is sometimes negligible compared with the other effects of transport. This is the case for heterogeneous permeability fields like the classical multi-Gaussian lognormal fields for which the transition between high and low flow zones remains gradual enough Salamon et al. [2006]. While not fully tested, this point was hinted in previous studies Tompson and Gelhar [1990]. In these conditions of heterogene- 
ity, random walkers are much more dispersed by the velocity field and its gradient within the mesh cells than by discontinuities at the cell interfaces.

At the other extreme, dispersive effects of discontinuities are critical for the sharp transitions of velocities tangent to the main flow direction Uffink [1985]. The first methods for handling the dispersion discontinuity were based on reflection principles approximating the complete solution to the discontinuous interface, which can be obtained through the method of images. Bechtold et al. [2011], Hoteit et al. [2002b], Semra et al. [1993], Uffink [1985]. When crossing the interface, random walkers are either reflected or transmitted. Starting from the higher dispersion side, random walkers are more likely reflected than dispersed, thus preventing from spurious accumulation of particles in the lower dispersive side. While conceptually simple, these methods raise implementation and performance issues. In multidimensional domains, random walkers can be reflected by several close interfaces, especially in the vicinity of cell corners. In low flow zones, random walkers may remain close to the interface and cross it numerous times, with the consequence of slowing down the algorithm.

TDRW methods are in essence more appealing than RW because their random walkers stop at the interface before being transferred to neighboring cells Bodin et al. [2007], Delay and Bodin [2001]. The time of flight to the interface is drawn from exact or approximate analytical solutions to transport in homogeneous media. Developed first in one-dimensional media for simulating solute transport in fracture segments Bodin et al. [2003], Painter and Cvetkovic [2005], TDRW have been extended to multidimensional media Bodin [2015], Delay et al. [2002]. When local analytical solutions of the travel time distribution can be obtained, TDRW methods are highly efficient to couple the advective and diffusive/dispersive transport processes. In the absence of local analytical solutions, alternative methods have been developed as a mix between "space-domain" random walk (classical random walk) and time-domain random walk and trying to combine both the spatial and temporal advantages. The constant displacement scheme consists in choosing a spatial scale $\Delta x$ much smaller than the mesh scale 1 and deriving the time step $\Delta t$ from the spatial scale divided the local velocity $\mathrm{v}$ Beaudoin et al. [2007], Wen and Gomez-Hernandez [1996], de Dreuzy et al. [2007]: $\Delta t=\Delta x / v$. The classical space-domain random walk method is then run once for this time step $\Delta t$ using both advection and dispersion mechanisms. The control by the velocity at the location of the random walker ensures that the mean displacement remains always equal to $\Delta x$. The characteristic number of steps taken within the mesh cell $\mathrm{n}$ can be obviously fixed by choosing $\Delta x=l / n$. The varying time step $\Delta t$ according to the location in the domain controls evenly over the whole domain the balance between accuracy and efficiency. This method combines some of the efficiency of the TDRW method by fixing the mean displacement and the generality of the classical Space-Domain RW. It also fundamentally respects the flow structure within the mesh cell because the small displacements correctly follow the structure of flow lines in each cell. In classical implementations, dispersion discontinuities treated by the reflection method is again an issue. It could be solved by switching to TDRW when the random walker comes close enough to one of the cell facets. Close to cell corners, only the interface in the direction of the velocity could be considered. In terms of implementation, RW and TDRW methods are interesting because they are based on a double particle and space decoupling. Random walkers are independent from each other and their behavior remains local. 
Except for velocity gradient discontinuities at interfaces, random walker motions do not need any other information than the mesh cell properties. The implementation can be made generic to mesh structures by specifying the transport operator at the mesh cell scale. Performances can be enhanced with memory-distributed as well as computation-distributed parallel methods. Memory-distributed methods are appropriate to large computational domains that cannot be stored on the hardware managed by a single processor Beaudoin et al. [2006, 2010], Beaudoin and de Dreuzy [2013], Kaludercic [2004]. Computation-distributed methods are used for smaller grids when high accuracy requires a huge number of particles Barker et al. [2012]. Even though implementation costs can be significant, they might be factorized to any mesh cell type simply because the specificity of the mesh only appears locally when tracking the random walkers within the mesh cells.

\subsection{Purely diffusive issues}

In the case of purely diffusive issues, $\mathbf{u}(\mathbf{x})=0$, we are led to solve

$$
\phi(\mathbf{x}) \frac{\partial c(\mathbf{x}, t)}{\partial t}=\nabla \cdot(D(\mathbf{x}) \nabla c(\mathbf{x}, t))+s(\mathbf{x}, t)
$$

In the hydrogeology practice, or in the oil and gas industry, $D(\mathbf{x})$ is the diffusivity given by $D(\mathbf{x})=\frac{k(\mathbf{x})}{\mu c_{t}}, \mu$ is the fluid viscosity and $c_{t}$ the total compressibility of the medium (rock plus fluid). A source term in the form $s(\mathbf{x}, t)$ representing either sources and sinks, or boundaries has been added in the right hand side of the equation. Note that the equation can describe either tracer, thermal or pressure diffusion in a porous medium of weak compressibility. The linearity of this equation allows us to consider the cases where $s(\mathbf{x}, t)$ is in the form $\delta(t) \delta(\mathbf{x}-$ $\left.\mathbf{x}_{0}\right)$. This means that we are interested in the Green's function of the previous equation. To go farther, a first step is to represent properly the heterogeneous (or fractured) medium by means of a geometrical meshing. In a second step, using a suitable numerical scheme results in a discrete set of equations to be solved. Finally, RW methods are used to solve the equations and to glance at some information concealed in these equations.

\subsubsection{Discretisation of heterogeneous and fractured media}

A discretization of the medium can be obtained via any available mesh builder handling triangular or quadrangular elements (or the corresponding elements in the three-dimensional case). In the case of bulk heterogeneities such as those generated by geostatistical methods, any sufficiently refined meshing compared with the underlying correlation length will capture most of the heterogeneities 3.3.1.

In the case of sharp heterogeneities enclosing discontinuities such as between different layers, it is assumed that the meshing follows the discontinuities. In the case of densely fractured media, building conform meshes (i.e. to make it simple not too much distorted) closely following the discrete network of fractures becomes quite infeasible 3.3.1. 


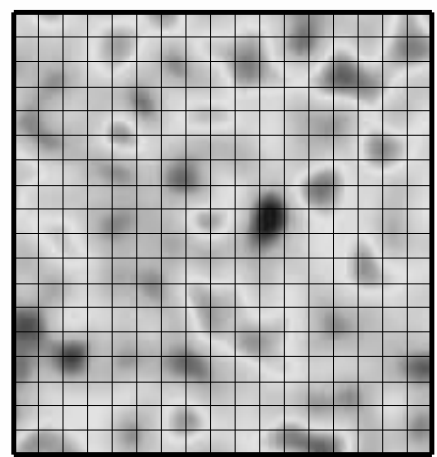

Fig. 1 An heterogeneous medium and the associated mesh

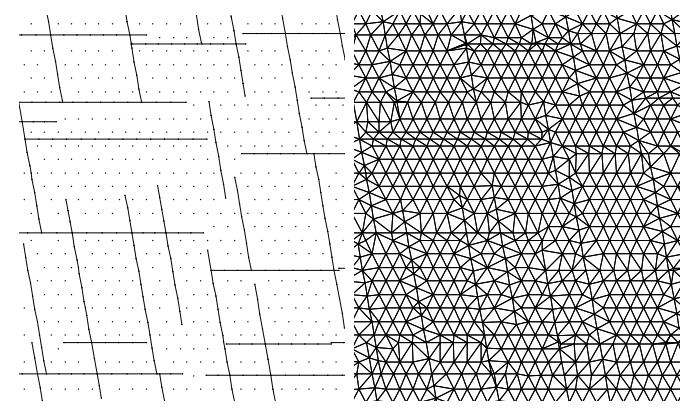

Fig. 2 A fractured unit cell and its triangulation

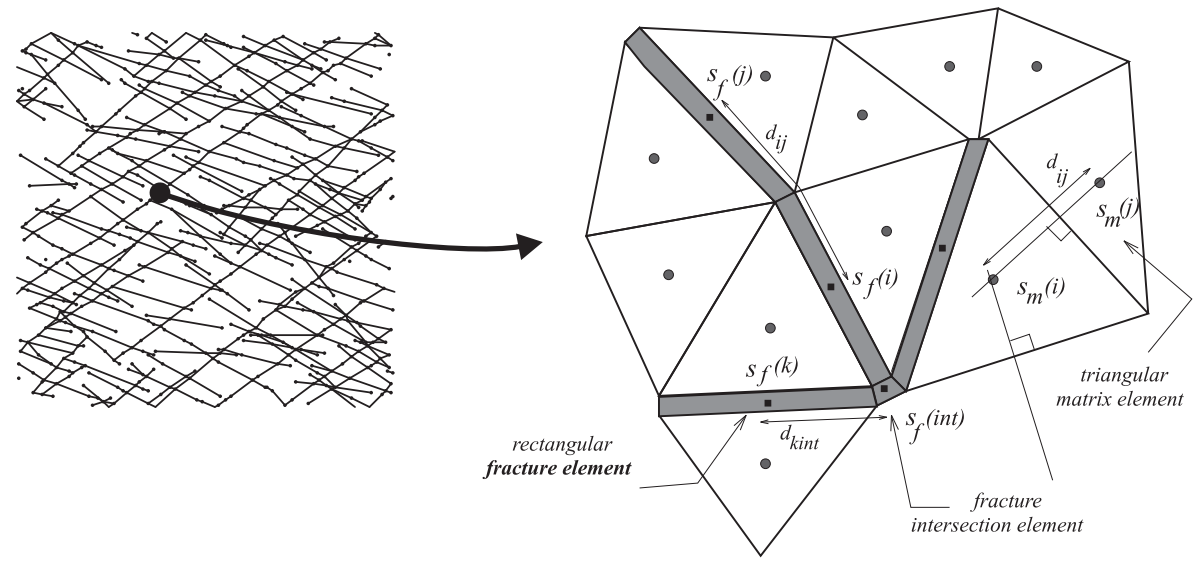

Fig. 3 Local zoom of the mesh 
In the second step, the diffusion equation (42) can be discretized using Finite Volume or Finite Element methods, yielding usually a linear differential set of equations in the form of:

$$
\begin{aligned}
V_{i} \phi_{i} \frac{\partial p_{i}(t)}{\partial t} & =\sum_{j \text { neighbor } i} T_{i j}\left(p_{j}(t)-p_{i}(t)\right) \\
& +\int_{\text {grid block } i} s(\mathbf{x}, t) d \mathbf{x} .
\end{aligned}
$$

This discretizing step can result in additional geometrical constraints for the meshing that are beyond the scope of this study. Using for example a Finite Volume scheme on a Cartesian regular grid of spatial step $\Delta x$, the discretized version of this equation in $D$ dimensions for each grid block of size $(\Delta x)^{D}$ can be written as:

$$
\begin{aligned}
(\Delta x)^{D} \phi_{i} \frac{\partial p_{i}(t)}{\partial t} & =\sum_{j \text { neighbor } i} T_{i j}\left(p_{j}(t)-p_{i}(t)\right) \\
& +\int_{\text {grid block } i} s(\mathbf{x}, t) d \mathbf{x} .
\end{aligned}
$$

The summation is carried out with respect to index $j$ labeling the nearest neighbors of the current grid block $i$.

It remains to define the so called transmissivities or inter block conductivities $T_{i j}$ between the two adjacent grid blocks $i$ and $j$ with respect to the underlying heterogeneous map. A classical choice is the harmonic mean given by:

$$
T_{i j}=2 \frac{d_{i} d_{j}}{\left(d_{i}+d_{j}\right)}
$$

where subscript $i$ indicates that the values are evaluated at the center $\mathbf{x}_{i}$ of the i-th grid block. This provides a consistent discretization of (43) ([Carthy, 1993a,b, 1991, 1990]). In the particular case of a fractured porous medium, mixing hydraulic properties of fractures and matrix, a different choice is usually taken by assigning diffusion coefficient to meshes $i$ in the form:

$$
d_{i}=\left\{\begin{array}{ll}
d_{m}, & \text { if } \mathbf{x}_{i} \in \Omega_{m} \\
d_{f}, & \text { if } \mathbf{x}_{i} \in \Omega_{f}
\end{array}\right\}
$$

Here, $\Omega_{f}$ and $\Omega_{m}$ represent the fracture and the matrix domains, respectively. Using a sufficiently refined grid, allows to rely upon standard Euler time discretization and solvers to produce accurate solutions of the discretized version of (43)

It is noteworthy that many discretization methods yield linear systems in the form of (43). Combining various mesh generation methods with compatibles discretization schemes for the diffusion operator will always render the same general form of the discretized equations, the latter simply changing by the stencil of neighbor cells involved in each equation and the formulas of inter-block transmissivities(45). A rather complete analysis of this last issue can be found in Romeu and Noetinger [Romeu and Noetinger, 1995]. It was observed that convergence to the solution of the continuous equations can be greatly accelerated according to the meshing and discrete scheme used. More recently, Wang et al [Wang et al., 2014, Qu et al., 2014] detailed the local flow field close to the corners between adjacent grid blocks. These authors were able to justify the general power law averaging as the rule for generating inter-block coefficients by using the so called "finite analytic approach". It must be kept in mind that in the case of 
extreme contrast, the standard choice of harmonic means (45) can provide severe underestimations of the large scale diffusion, because the harmonic mean is mainly controlled by the smallest value. In the case of percolation lattices involving highly contrasted properties, the percolation threshold might be artificially shifted. In the sequel, we will no longer come back on the choice of inter-block parameters even though it is an important feature for solving flow and transport equations.

\subsection{Random walk methods for dual-porosity models}

Fractured media are generally made of highly conductive fractures present at several scales and embedded in a low permeability matrix. This high contrast of properties led to the introduction of dual-porosity models where matrix and fractures are treated separately and coupled through an exchange term. These models have been used to simulate various processes, such as fluid flow [Barenblatt and Zheltov, 1960, Warren and Root, 1963], solute transport [Dershowitz and Miller, 1995, Cvetkovic et al., 2004], and electric current flow [Roubinet and Irving, 2014], and they can also be associated with Discrete Fracture Network (DFN) approaches to consider the geometrical complexity of realistic fracture networks [Cacas et al., 1990, Delorme et al., 2013a]. In the purely diffusive case, these models can be ruled by the following equation:

$$
\left(\phi_{f} V_{f} \delta(t)+\phi_{m} V_{m} f(t)\right) * \frac{\partial c(\mathbf{x}, t)}{\partial t}=\nabla \cdot(D(\mathbf{x}) \nabla c(\mathbf{x}, t))+s(\mathbf{x}, t) .
$$

Here, $V_{f}$ and $V_{m}$ denote the volume fractions of the fractures and the matrix, respectively, and $\phi_{f} \phi_{m}$ are the associated porosity. Introducing the Laplace transform defined by ${ }^{1}$

$$
g(s)=\int_{0}^{+\infty} \exp (-s t) g(t) d t
$$

The Laplace transform of eq (47) yields:

$$
\left(\phi_{f} V_{f}+\phi_{m} V_{m} f(s)\right)(s c(\mathbf{x}, s)-c(\mathbf{x}, t=0))=\nabla \cdot(D(\mathbf{x}) \nabla c(\mathbf{x}, s))+s(\mathbf{x}, s) .
$$

In some dual-porosity models, RW methods have been used for: (i) up-scaling the transfer properties required to describe the fracture-matrix interactions, (ii) directly solving the considered flow or/and transport problem, and (iii) evaluating the flow or/and transport effective properties. In this section, we describe a few of these applications distinguishing the dual-porosity models based on a continuous representation of the fractures (Section 3.5) from models based on an explicit representation of the DFN (Section 3.5.1).

\subsection{Continuum dual-porosity models}

Solving fluid flow in fractured porous media can be performed by resorting to a continuous dual-porosity model, such as the one described in Appendix B. The model description handled in the present study lets appear transfer functions and

\footnotetext{
1 In order to simplify notations, functions with "s" variables correspond to Laplace transforms throughout the rest of the paper.
} 
coefficients between fracture and matrix continua, denoted $f(t)$ and $\lambda$, respectively. Although these functions and coefficients can be determined analytically for simple cases (expressions (71)-(72)), a numerical evaluation is required for complex cases. In this context, Noetinger and Estebenet [2000], Noetinger et al. [2001a,b], Landereau et al. [2001] applied directly a TDRW method (named CTRW in their papers) to determine these transfer parameters. The key observation Noetinger and Estebenet [2000], Noetinger et al. [2001a] is that $f(t)$ can be interpreted as being the probability density function (pdf) of the first exit time distribution of a particle initially launched from a random location, then undergoing a random motion in the matrix and finally exiting in the fracture domain at the matrix boundary. This function $f(t)$ may also be identified as the local memory introduced for general mobile-immobile physical and chemical interaction processes Carrera [1998], Willmann [2008]. The following expansion, which can be verified explicitly in the few analytical solutions of $f(s)$ provided in the appendix B can be derived Noetinger and Estebenet [2000], Noetinger et al. [2001a]:

$$
f(s)=1-\frac{\phi_{m} V_{m}}{\lambda} s+o(s) .
$$

Recasting the equation in the real domain yields $\langle t\rangle=\int_{0}^{+\infty} t f(t) d t=\frac{\phi_{m} V_{m}}{\lambda}$ Therefore, the mean residence time of random walkers in the matrix is directly related to the transfer coefficient introduced in double porosity models. It is possible to show mathematically that the $\lambda$ coefficient arising from this definition exactly coincides exactly with the coefficient obtained from large scale averaging or homogenization theory Noetinger et al. [2001b]. These considerations result in the following algorithm that provides a full determination of the exchange function $f(t)$ as well as the parameter $\alpha$. Without loss of generality, we assume $\phi_{m}=1$.

1. choose the number $\mathrm{N}$ of independent particles.

2. To start the i-th particle, choose one matrix node $j_{i}$ with a probability proportional to the volume of the associated grid block, $\mathrm{k}=1$.

3. While the particle has not left the matrix, at step $\mathrm{k}$ of the algorithm, displace the particle from the site "i" to its nearest neighbor site "j", with a probability equal to $b_{i j}$.

4. Update the time counter with the relation:

$$
t_{k}=t_{k-1}-\frac{V_{i}}{B_{i}} \log (\text { rand }) .
$$

Here, rand stands for a random number picked from a uniform distribution over $[0,1]$.

5. If the particle enters a grid block belonging to the fracture region at iteration $\mathrm{k}$, store the exit time $t^{i}=t_{k}$ of the current particle $\mathrm{i}$ and launch the $\mathrm{i}+1$ th particle.

6. Store the $\mathrm{N}$ independent exit times $t^{(1)}, \ldots, t^{(N)}$

7. End.

It is thus easy to obtain an estimation of the histogram of the distribution yielding $f(t)$. The quantity $\lambda$ can be estimated by means of the relation

$$
\lambda=\frac{V_{m}}{\left\langle t_{m f}\right\rangle} \simeq \frac{V_{m}}{\frac{1}{N} \sum_{i=1}^{N} t^{(i)}}
$$


Therefore, the above algorithm returns a direct determination of the complete exchange function; it is very fast and efficient because there is no need of iterations (particle motions) in the fractures. The algorithm was successfully tested on simple geometries consisting of a parallel set of fractures and provided good comparison with existing analytical determinations of both $f(t)$ and $\lambda$, fig 3.5. Further tests on simple DFN's with some randomness in the fracture characteristics were also carried out.
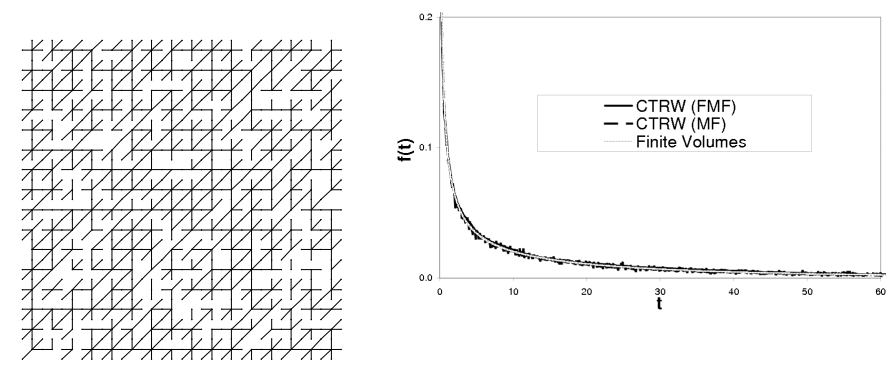

Fig. 4 Left: a fractured medium. Right: transfer function by TDRW and by finite differences.

It remains to characterize transfer functions for complex DFN's. Finding generic analytical forms generalizing the equations $(70,71,72)$ and allowing us to account for very wide distributions of matrix block size remains to be done. At short times, corresponding to large Laplace parameter $s, f(t)$ function is sensitive to the fracture's surface to volume ratio. At larger times, $f(t)$ samples the whole block size distribution, making that some fractal and thus "anomalous" power law behavior may be anticipated. This could lead in the real time domain to anomalous transfer kernels equivalent to fractional derivatives Néel et al. [2011, 2014]. There is no doubt that the preceding TDRW method will be useful to feed the investigations with numerical results. Figure 3.5 shows a realization of a percolation lattice with a proportion $p=0.57$ of active fractures. In figure 3.5 , we plotted the dependence $p-p_{c}$ of the mean residence time in the matrix (of diffusivity one unit) with respect to the proportion of active fractures. The different curves correspond to different mean residence times $\langle t\rangle$ that depend on the fracture subnetwork that was kept, i.e., all the fractures including non-relevant isolated clusters, or only the percolation backbone. Intermediate curves correspond to different treatments of the remaining clusters. One can note that keeping the whole set of fractures (red dots) in 3.5 does not lead to any critical divergence of the mean residence time close to $p_{c}$. Further studies should be carried out to get a better characterization of the associated critical exponents.

Note that random walk methods have also been used in continuum dualporosity models to directly model transport in fractured porous media [Liu et al., 2000, Pan and Bodvarsson, 2002a]. In the previous studies, advection-dispersion equations are considered in the fracture and matrix continua, and a probability of transfer between these two continua is defined analytically. 


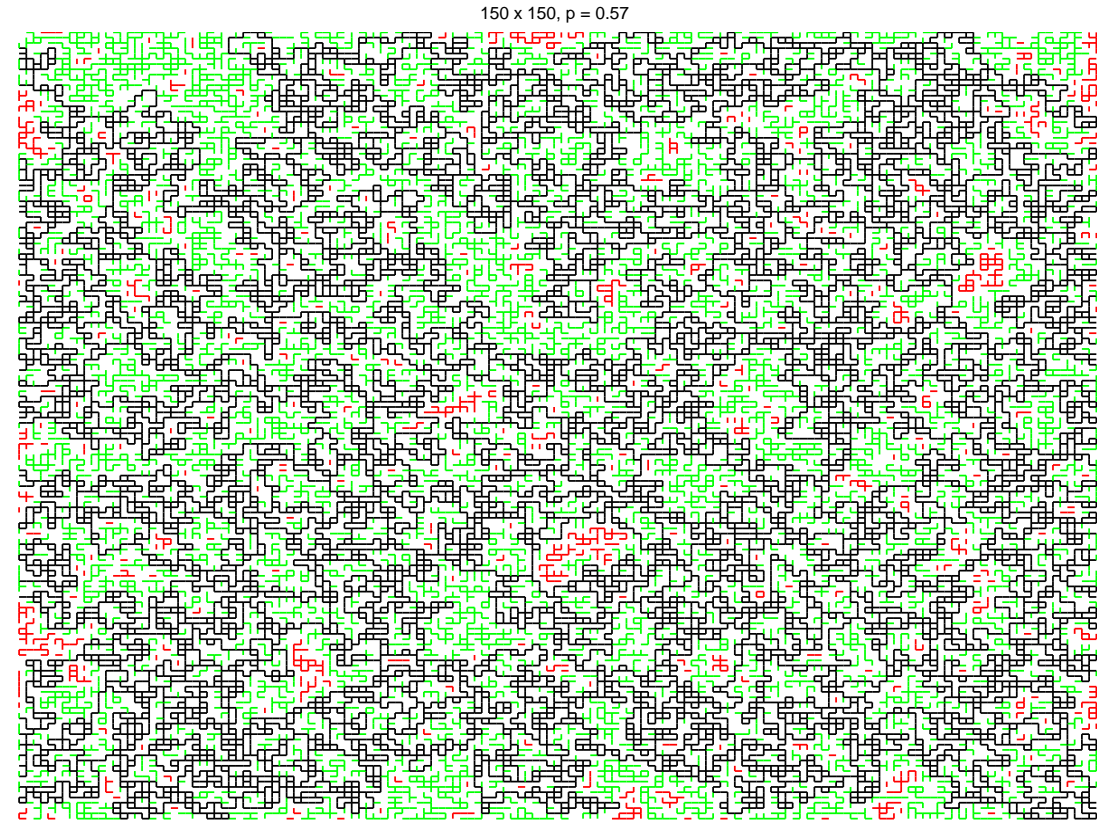

Fig. 5 A percolation network $\mathrm{p}=0.57$, red $=$ isolated clusters, black $=$ backbone, green $=$ dead-ends

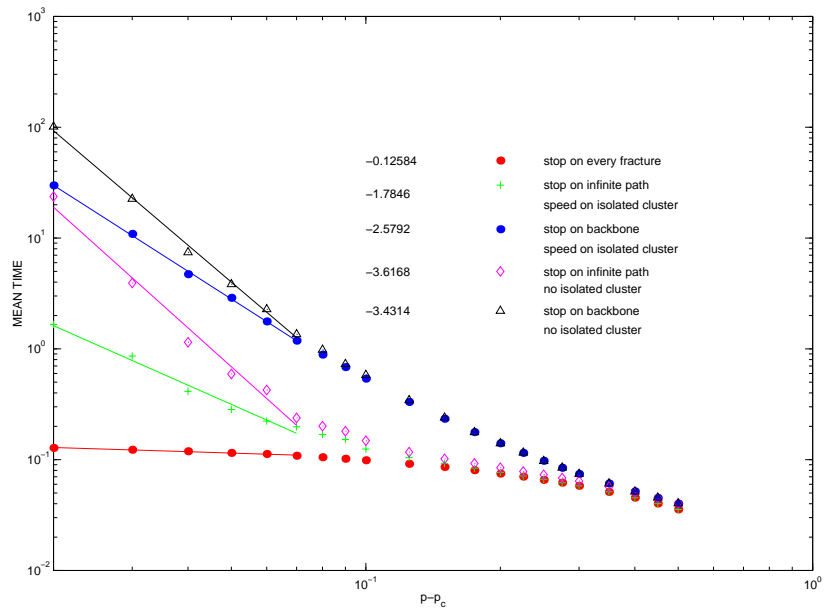

Fig. 6 Exchange coefficient computed for the percolation network $p=0.57$

\subsubsection{Discrete dual-porosity models}

In the preceding paragraph, it was shown that the transfer function $f(t)$ could be determined efficiently using TDRW. Once $f(t)$ is found applications can be envisioned by using the double porosity equations (61) or the alternative form (64). Another option is to keep the details of the DFN explicitly. Some studies by Chang and Yortsos Chang and Yortsos [1990], Acuna and Yortsos Acuna and 
Yortsos [1995] were carried out in this spirit using two-dimensional DFN close to the percolation threshold. The matrix to fracture flow was ignored. A threedimensional generalization and a solution to include matrix to fracture flow was proposed in Noetinger and Jarrige [2012],noetinger2015. By introducing the set of fracture intersections labeled by $j$, and denoting $P_{j}$ as the average pressure on the $\mathrm{j}$-th intersection, it is possible to derive the following set of equations:

$$
\forall i=1, N_{\cap}, \quad \sum_{j \in J(i)} K_{i j}^{11}\left(V_{f} \delta(t)+V_{m} f(t)\right) * \frac{d P_{j}(t)}{d t}=\sum_{j \in J(i)} V_{f} T_{i j}^{11} P_{j}(t) .
$$

To simplify notations, both matrix and fracture porosity are here equal to unity. The quantities $K_{i j}^{11}$ and $T_{i j}^{11}$ depend explicitly on the shape of the fractures and can be determined with fast algorithms Khvoenkova and Delorme [2011], Delorme et al. [2013b]. We identify a discrete form of the set of equations (64) that can be solved using TDRW with trapping as introduced by Dentz et al Dentz et al. [2012], equations (26) and (29) (30) and (31) of their contribution. Identification of the formalism by Dentz et al. and Eq. XX is obtained by stating

$$
\left(V_{f}+V_{m} f(s)\right)=\frac{1}{\mu(\mathbf{x}, s)}
$$

where $\mu(\mathbf{x}, s)$ is the local memory function introduced in Dentz et al. [2012]. To summarize this section, one can state that TDRW is useful to both:

- determine $\mathrm{f}(\mathrm{t})$ using random walkers in the matrix only, the DFN acting as a boundary

- once $\mathrm{f}(\mathrm{t})$ properly known, solving equations 51 to up-scale the DFN.

TDRW methods associated with an explicit representation of the DFN have also been used to model transport processes in fractured media. Some models only consider advective displacement in the fractures with the aim of studying hydrodynamic dispersion in two-dimensional heterogeneous percolation networks [Rivard and Delay, 2004]. In these studies, binary and log-normally distributed hydraulic conductivity fields are considered, and the impact of this heterogeneity on the longitudinal dispersion coefficient is analyzed at the percolation threshold. In other models, advection-diffusion mechanisms in the fractures and pure diffusion in the matrix are considered, with sometimes a mesh-free representation of the matrix. This dual-porosity formulation enables one to reduce the numerical cost and the algorithm complexity related to the meshing step of each simulation. With these techniques, particles only move in the fractures and their diffusion into the surrounding matrix is considered via an additional retardation time [Cvetkovic et al., 2004, Dershowitz and Miller, 1995, Roubinet et al., 2010]. When this retardation time is deduced from analytical solutions, it relies on specific physical and geometrical assumptions. For example, Dershowitz and Miller [1995] consider a pure-diffusion equation in matrix blocks of regular shape, whereas Cvetkovic et al. [2004] make use of retention models based on analytical solutions of fracture-matrix systems (e.g., Tang et al. [1981]). In the latter case, the matrix surrounding each fracture is assumed infinite with the meaning that matrix diffusion is not limited by the presence of nearby fractures. To overcome this limitation, Roubinet et al. [2010] add the possibility for each particle to transfer from one fracture to another 
by diffusion through the matrix blocks. The method is applicable to heterogeneous fractured porous media without restriction on the matrix block geometry or network density.

The numerical methods previously described have been used to evaluate upscaled transport properties and to analyze the impact of structural heterogeneity on the large-scale transport behavior of fractured media. In Cvetkovic et al. [2004], Painter and Cvetkovic [2005], Painter et al. [2008], up-scaled transport properties are first evaluated over small discrete fracture networks, and then used to perform large-scale simulations. This efficient two-step method has been applied to the Äspö Hard Rock Laboratory in Sweden [Cvetkovic et al., 2004, Painter and Cvetkovic, 2005] and has been extended by accounting for first-order (kinetics) transformation [Painter et al., 2008]. Other RW methods associated with a DFN representation have helped to understand the impact of DFN heterogeneity on large-scale effective transport properties. More precisely, Liu et al. [2007] focus their study on the dependence with space scale of the effective matrix diffusion coefficient deduced from field tracer experiments. This scale effect has been observed in numerous characterization studies of fractured rocks, and the numerical simulations conducted in Liu et al. [2007] showed that this effect was related to the DFN heterogeneity. Finally, the impact of DFN heterogeneity has also been studied in Roubinet et al. [2013] by way of numerical transport simulations conducted over synthetic fracture networks with large ranges of hydraulic properties and matrix block size distribution.

\subsection{Measuring Mixing Properties From Random Walk Simulations (Tanguy)}

Mixing is one of the key properties of interest when studying transport and reaction phenomena in heterogeneous porous and fractured media [Kitanidis, 1994, Dentz, 2011]. While dispersion measures the spatial extent of transported plumes, mixing quantifies the distribution of concentration of dissolved chemical species transported in the fluid phase [Borgne and T., 2015]. As such, it measures both the dilution of plume as it mixes with the resident fluid and the probability of dissolved reactive species to meet and react [de Simoni, 2005]. Different mixing measures have been proposed including the dilution index [Kitanidis, 1994], the intensity of segregation [Danckwerts, 1952] and the scalar dissipation rate [Ottino, 1989]. The latter measures the rate at which concentration gradients are dissipated under the combined action of advection and diffusion. It can be directly translated into an effective reaction rate when considering fast reactions [Le Borgne T., 2010, de Simoni, 2005].

Numerical particle tracking methods are particularly useful to investigate dispersion and mixing as they avoid numerical dispersion. However, they are typically characterized by a significant noise in concentration gradients, whose reduction requires using a large density of particles. This is an issue to estimate scalar dissipation rates, which is expressed in terms of concentration gradients as,

$$
\chi(t)=\int d x D[\nabla c]^{2} .
$$

where $D$ is the local diffusion coefficient and $c$ is the local concentration. This measures of mixing is significantly affected by errors in concentration gradients 
since it depends on the local concentration gradient squared. However, for finite time injection conditions, i.e. when no additional solute is injected in the domain when scalar dissipation is measured, one can show that the scalar dissipation rate is directly linked to the temporal derivative of the concentration squared as,

$$
\chi(t)=-1 / 2 \frac{d}{d t} \int d x c^{2} .
$$

This expression shows that the scalar dissipation rate is a measure of the decay of concentration variance as the transported plume is diluted in the resident fluid. As illustrated in figure 3.6 and discussed in Le Borgne T. [2010], it turns out that expression 54 is much less sensitive to numerical noise than the classical expression for scalar dissipation rate (equation 53). This is because it does not require evaluating concentration gradients. Based on this method, mixing can be evaluated efficiently from random walk particle tracking simulation in heterogeneous media Le Borgne T. [2010]. As illustrated in Figure refFig:scalardissipationrated, heterogeneity in permeability is found to induce temporal scalings of the mixing rates that depart from those expected for homogeneous media. This phenomenon can be explained by an enhancement of diffusion to due plume stretching by velocity gradients [Le Borgne et al., 2013].

4 Application from pore to field scale

\subsection{Effective flow and transport parameters}

A direct application of RW methods is the determination of effective conductivity (or diffusivity) of heterogeneous media, by means of Einstein relation relating mean square displacement to the effective coefficient of the diffusion equation (Einstein [1956]):

$$
R^{2}(t)=\left\langle(x(t)-x(t=0))^{2}\right\rangle=2 d D(t) t .
$$

where $d$ is the dimensionality of the system and $D(t)$ denotes the scaling coefficient of the diffusion equation under consideration (diffusion, diffusivity or conductivity). In the case of bulk heterogeneous media, it is possible to show that the convergence of $D(t)$ to its asymptotic value $D_{\text {eff }}$ is reached once $R^{2}(t) \gg l_{c}$, where $l_{c}$ is the permeability correlation length. Physical considerations show that at short times, $R^{2}(t) \sim \bar{D} t$ where $\bar{D}$ denotes the average diffusivity, because the diffusive particles sample local homogeneous regions of size $l_{c}$. The cross-over between both regimes can provide information of the REV size. This technique has been also often used to analyze binary media, for instance obtained from X-ray tomographic images (e.g. Gouze and Luquot [2011]) and measure the effective diffusion and tortuosity from the asymptotic regime but also to evaluate the surface to volume ratio from the transient regime (P.N. Sen and Halperin [1994], Sen [2003, 2004]).

\section{CAN MAKE A FIGURE FOR ILLUSTRATION HERE IF NEEDED.}

RW calculations of sample scale effective properties of binarized images of porous media can be efficiently performed using TDRW approach were the lattice defining the fixed distance jump of the particle is mapped on the image voxel. Calculation of $D_{\text {eff }}$ from binarized media can be performed by solving a boundary value problem, mimicking a diffusion experiment, were particles are set at one 


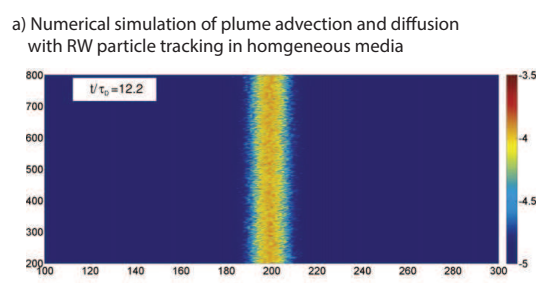

c) Numerical simulation of plume advection and diffusion with RW particle tracking in heterogeneous media

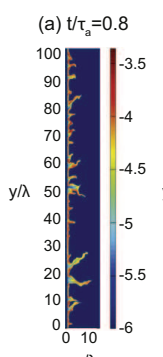

(b) $\mathrm{t} / \mathrm{r}_{\mathrm{a}}=2.6$

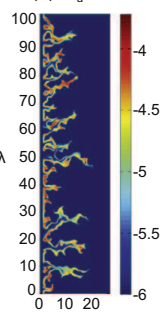

$\mathrm{x} / \lambda$ (c) $\mathrm{t} / \mathrm{r}=8.2$

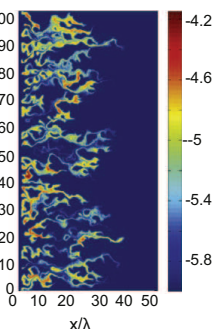

b) Temporal evolution of the scalar dissipation rate in homogeneous media

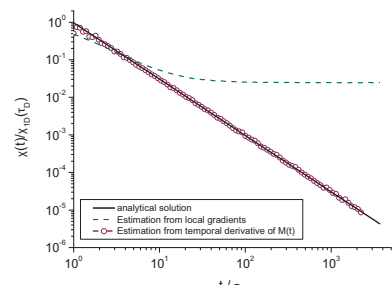

d) Temporal evolution of the scalar dissipation rate in heterogeneous media

Fig. 7 a. Numerical simulation of the transport of a line of tracer in a homogeneous media under advection and diffusion with particle tracking. $\tau_{D}$ is the characteristic diffusion time over a pixel of size $\Delta x, \tau_{D}=\Delta_{x}^{2} / D \mathrm{~b}$. Estimation of the scalar dissipation rate from the local concentration gradients 53 and from the local concentration squared $M(t)=\int d x c^{2} 54$. The latter compared much better than the former to the analytical solution for a homogeneous media $\chi_{1 D}(t)=\frac{C_{0}^{2} L_{y} t^{-3 / 2}}{8 \sqrt{2 \pi D}}$. c. Numerical simulation of the transport of a line of tracer in a heterogeneous media under advection and diffusion with particle tracking. d. Temporal evolution of scalar dissipation rates estimated for different permeability field variances. Heterogeneity in permeability affects the temporal scaling of the scalar dissipation rate and thus the global mixing rate. Adapted from Le Borgne T. [2010].

boundary and the first passage time monitored at the opposite boundary, but a more efficient method is to distribute the particle randomly in the pore space (initial value problem) and apply directly (55) to measure $D(t)$ versus time $t$ and obtain $D_{\text {eff }}$ from the asymptotic behavior, i.e. at very long times when the particle motion has averaged over several pores. Practically, it is sometimes difficult to obtain an asymptotic behavior meaning that the sample do not display a finite scale heterogeneity or that computations has been stopped to early. For instance particles may spend a lot of time to reach tiny connected paths in binary media close to the percolation threshold and anomalous diffusion may be observed Sahimi [2011], Superdiffusive [1989].

Similarly, RW methods have been used to calculate the effective dispersion of binarized porous media solving (5) where the steady-state fluid velocity field is usually obtained solving the Navier-Stokes equation using standard methods such as finite volume. The geometry of the simulations as well as the boundary and initial conditions are generally chosen to mimic laboratory tracer tests through core, i.e. using permeameter-like conditions were a passive tracer is injected at one side and the tracer breakthrough curve (BTC) monitored at the opposite side. However pulse injection is usually applied because of it permit much faster computations (using less particles) than the constant concentration boundary condition 

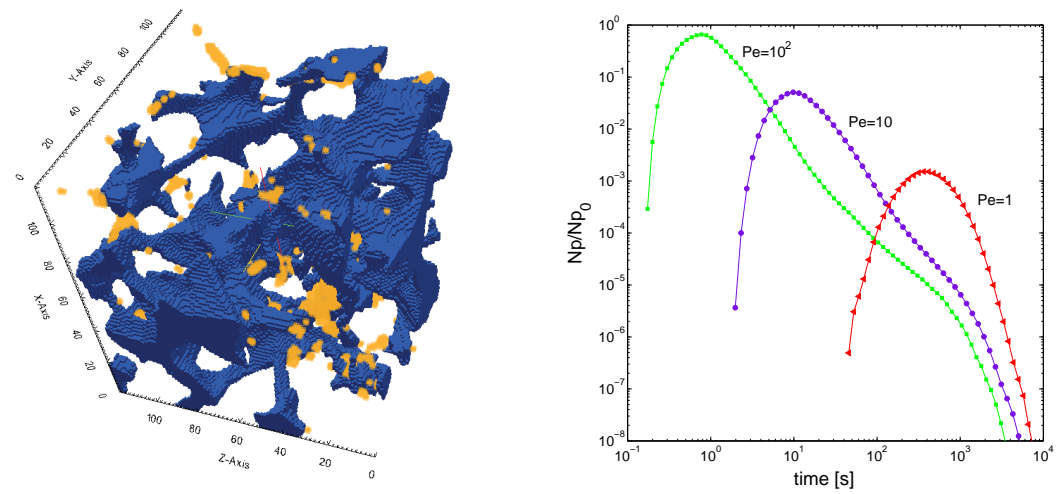

Fig. 8 Left: three-dimensional visualization of the pore network (blue) and the connected microporosity (yellow) for a sub-volume of $120^{3}$ voxels of the $\left(0.8 \mathrm{~mm}^{3}\right)$ Berea sandstone sample studied by Gjetvaj et al. [2015]. Right: BTCs for pore-scale mobile-immobile transport through the Berea sample for different values of the Peclet number computed by TDRW.

usually applied experimentally. In general the size of the domain is bounded by the computational limit for solving the Navier-Stokes equation and therefore restricted to $3 \mathrm{D}$ lattices of size $300^{3}$ to $1000^{3}$ that correspond to millimeter-sized rock samples. Nevertheless this approach is really attractive to study the relations between the flow field properties and the the dispersion which is usually characterized by marked non-Fickian BTCs. Bijeljic and co-authors (Bijeljic B. and Blunt [2013a,b]) studied the dispersion in carbonate and sandstone rock samples using binarized X-ray tomographies. They simulated transport by moving particles by advection along streamlines (previously computed from the flow field) and using a random walk method with constant particle jump time to describe the molecular diffusion. The authors characterized the heterogeneity in term of a throat size distribution and results showed that an asymptotic behavior is observed when particles visited many throats. Conversely, Gjetvaj and co-authors (Gjetvaj et al. [2015]) investigated the origin of non-Fickian transport using Berea sandstone containing a low fraction of micro-porous cement with pore size below the resolution of $\mathrm{X}$-ray microtomography technique (Figure 8). They showed that this micro-porous phase can be conveniently described as an immobile diffuse domain while the solute transport in the resolved macroporosity (the mobile domain) results from the combination of Navier-Stokes flow and diffusion. Here the transport of the tracer in both the mobile and immobile domains is modeled using the TDRW approach. The resulting BTCs for different values of the Peclet number displayed markedly non-Fickian behaviors triggered by the flow field heterogeneity and the diffusion in the heterogeneous microporosity. Finally the authors proposed an upscalled 1D CTRW model that fit the BTCs obtained from the pore scale computations; the 1D CTRW simulations were also performed using a TDRW approach were the velocity heterogeneity is modeled by a truncated power-law whose characteristic time scales are related to the characteristic transport times in the mobile zones and the anomalous diffusion in the microporous phase is modeled as particle trapping events whose occurrence follows a Poisson process proportional to the mobile transition time and trapping time follows a truncated power-law. 
McCarthy Carthy [1993a,b, 1991, 1990] was the first to carry out TDRW techniques for conductivity up-scaling applications with the computation of large-scale equivalent permeability. More recently, random walk methods have also been used to up-scale the transport properties of heterogeneous porous media at several scales. For example, at the laboratory scale, CTRW methods can be associated with pore network models to evaluate the effective longitudinal dispersion of rock samples and to analyze the impact of advection and heterogeneity on this property [Bijeljic et al., 2004, Bijeljic and Blunt, 2006]. In these studies, the heterogeneity is considered in terms of a throat size distribution computed for Berea sandstone, and the results show that an asymptotic behavior is observed only after motion through many throats is experienced. At a larger scale, permeability heterogeneity has been represented with a lognormal exponentially correlated distribution and the flow equation has been discretized with a Finite Difference scheme [de Dreuzy et al., 2007, Beaudoin et al., 2010, Beaudoin and de Dreuzy, 2013]. In this case, the evaluation of large-scale effective longitudinal and transversal dispersion is performed by means of a two-dimensional [Beaudoin et al., 2010, de Dreuzy et al., 2007] and three-dimensional [Beaudoin and de Dreuzy, 2013] TDRW method. An additional advantage of TDRW methods for advective-dispersive transport in fractured networks is their capability to separate the transport within the fractures from mechanisms at fracture intersections. Different assumptions can be applied at the fracture intersections from complete mixing to streamline routing Berkowitz [1994], Kosakowski and Berkowitz [1999], Park [2003], resulting in markedly different mixing behaviors at the network scale Bruderer and Bernab [2001], de Dreuzy [2001], Park [2001]. Finally, the up-scaling of transport properties has also been subject to various attempts for fractured media, as described in Section 3.5.1.

\subsection{Interpretation of solute transport experiments}

At the laboratory scale, CTRW has been used to interpret transport experiments have been set up in either flow cells of porous media [Berkowitz et al., 2000], in fully or partially water saturated conditions, using passive or sorbing tracers [Bromly and Hinz, 2004, Cortis and Berkowitz, 2004, Hatano and Hatano, 1998], or in single fissures in granitic core samples using non-sorbing tracers [Jimenez-Hornero et al., 2005].

Random Walk approaches have been used to interpret field observation of non-Fickian properties obtained from in situ tracer experiments. One of the most common manifestation of non-Fickian transport processes is long time breakthrough curve tailing (Figure 4.2), that is the slow decay of tracer concentration at late times observed in many tracer breakthrough curves [Haggerty et al., 2000, Berkowitz et al., 2006]. This tailing behavior carries information on the underlying processes that induce non-Fickian transport.

For instance, matrix diffusion is known to impart a strong tailing. As explained in section 2, this problem can be modeled by in adding a sink-source term to the Fickian ADE to account for the mass transfers between the mobile domain and the matrix where transport is controlled by diffusion only (the immobile domain):

$$
\phi(\mathbf{x}) \frac{\partial c(\mathbf{x}, t)}{\partial t}-\nabla \cdot[\mathbf{D} \nabla c(\mathbf{x}, t)+\mathbf{v} c(\mathbf{x}, t)]+S(\mathbf{x}, t)=0
$$


with

$$
S(\mathbf{x}, t)=\phi^{\prime}(\mathbf{x}) \frac{\partial}{\partial t} \int d t^{\prime} M\left(\mathbf{x}, t-t^{\prime}\right) c\left(\mathbf{x}, t^{\prime}\right),
$$

where $\phi$ and $\phi^{\prime}$ denote the porosity in the mobile and immobile domain respectively and $M$ is the (possibly spatially distributed) memory function that contains all the information on the mass transfer process, the geometry and the volume fraction of the immobile domain as well as its accessibility to tracer particles issued from the mobile domain.

Formulations of this sink-source term $S(\mathbf{x}, t)$ to model the multiple rates nature of the mass transfers resulting from the transient diffusion in an homogeneous immobile domain of simple geometry were given by Haggerty and Gorelick [1995], Carrera [1998], Haggerty et al. [2000]. In this case tailing of the breakthrough curves decays as $c(t) \sim t^{-1.5}$.

However, natural systems often display dispersed clusters of matrix with heterogeneous diffusion properties (see for example figure 8) and different tailing of the breakthrough curve can be expected. Actually field observations report power law tailing in breakthrough curves such that $c(t) \sim t^{-1-\beta}$, with power law exponents spanning a broad range within $0<\beta<2$ [Haggerty et al., 2000] or with tailing displaying more than one slope such as the single-well injection withdrawal tracer tests published by Gouze et al. [2008a].

Gouze and co-authors (Gouze et al. [2008b]) computed the memory function $M(t)$ from X-ray microtomography images. First the authors processed the data in order to determine the interface between the mobile and the immobile domain and to evaluate the diffusion coefficient distribution inside the immobile domain. Then they compute the memory function using a constant time increment RW approach. The implementation of the calculation is as following. $N$ random walkers uniformly distributed over the mobile-immobile interface are released at the initial time $(t=0)$. The diffusion equation is solved by a discretized version of the Langevin equation (see (39)) and a specific procedure is applied to determine the trajectory of the random walkers through the heterogeneous pixelized matrix at each time increment. For $t>0$, the mobile-immobile interface is considered as an absorbing boundary, so the random walkers that jumps outside the immobile domain are removed. The total number of random walkers inside the immobile domain $N^{\prime}(t)$ is recorded until the last particle leaves the immobile domain. The $o p$. cit. authors showed that the memory function $M(t)$ can be obtained from the ratio $N^{\prime}(t) / N$. Then Gouze et al. [2008a]) compared the memory function computed at pore scale from the X-ray microtomography imaged rock sample to the memory function deduced from a set of field scale tracer tests in carbonates (Gouze et al. [2008a]) where the sample was cored. The authors concluded that the non-Fickian dispersion measured at meter scale is well explained by microscale diffusion processes in the matrix for this specific case.

Conversely, Le Borgne and Gouze [2008] developed a specific RW model to explain the two-slope tailing breakthrough curves measured by Gouze et al. [2008a]. The op. cit. authors implemented a continuous time random walk (CTRW) approach such as presented in section 2.2 in which the transition time distribution $\psi(t)$ was a dual-slope power law distribution. The model best fits the tracer test data with a transitional regime modeled by $\psi(t) \propto t^{-2}$ and an asymptotic regime corresponding the simple homogeneous double-porosity model (i.e. $\psi(t) \propto t^{-1.5}$ ). 
As alternatives to the heterogeneous diffusion modeled by multi-rate mass transfer or CTRW, different processes have been invoked to explain this range of exponents, including heterogeneous advection in independent flowpaths, modeled by stochastic streamtube approaches [Becker and Shapiro, 2003]. Both processes can be modeled by random walk approaches [Kang et al., 2015, Gjetvaj et al., 2015].

In the CTRW framework, a power law tailing $c(t) \sim t^{-1-\beta}$ can be modeled by considering a transition time PDF $\psi(t) \sim t^{-1-\beta}$. Transition to Fickian transport can be modeled by including a cut off in this power law [Dentz et al., 2004]. While CTRW approaches generally describe Non-Fickian transport a series a uncorrelated motions whose duration may be very long, stochastic streamtube approaches [Becker and Shapiro, 2003] assume that particle velocities are constant, hence fully correlated, along each streamline but differ from streamline to streamline. Becker and Shapiro [2003] have reported that this process leads to a characteristic breakthrough curve tailing $c(t) \sim t^{-2}$ under convergent tracer test conditions, and different exponents under push pull conditions, that depend on the push duration. Kang et al. [2015] have shown that both limiting processes can be modeled by considering a CTRW model with correlated transition times, whose correlation can be varied from zero to represent the CTRW model, to infinity to represent a stochastic streamtube model. It is not always easy to decipher the role of diffusive and advective processes as they can lead to similar power law exponents in breakthrough curves. As shown by Kang et al. [2015], this uncertainty on process controlling Non-Fickian transport can be resolved by combining tracer test experiments in different conditions, including cross borehole and push pull tests. Cross borehole tests are more sensitive to heterogeneous advection processes, while push pull tests are more sensitive to diffusive processes (Figure 4.2).

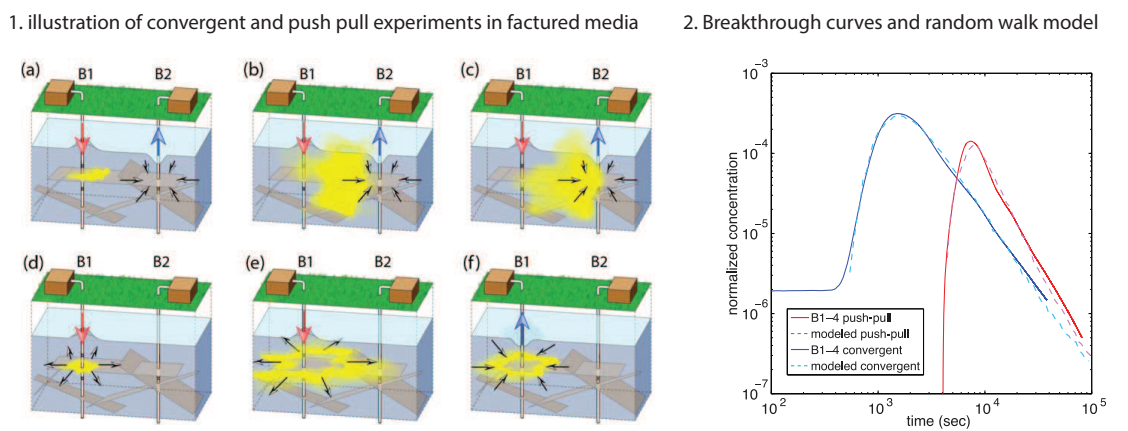

Fig. 9 1. Illustration of convergent and push pull tracer experiments in fractured media, from Kang et al. [2015], 2. Breakthrough curves measured at the Ploemeur site (H+ network) under convergent and push pull conditions, and random walk modeling (dashed lines) with a Markov Chain CTRW model, from Kang et al. [2015].

\subsection{Modeling of transport processes under ambient conditions}

RW methods have also been used to improve our understanding of transport processes under ambient conditions in natural environments. Numerous of these stud- 
ies have been conducted on fractured rock using TDRW methods within a discrete dual-porosity framework. Some of these models are described in Section 3.5.1 and have been used, for example, to describe the migration of radionuclides through fractured rock at the Äspö Hard Rock Laboratory in Sweden [Cvetkovic et al., 2004] and the Topopah Spring welded unit in Yucca Mountain [Pan and Bodvarsson, 2002b]. Other applications target the fractured Laxemar site in Sweden, which is part of the candidate repositories of spent nuclear fuel [Cvetkovic and Frampton, 2012], and the Kamaiski experimental mine site in Japan [Dershowitz and Miller, 1995].

Transport processes have also been modeled with RW methods in the context of colloidal transport in a shear zone [Kosakowski, 2004], chemical retention times in water catchments [Scher et al., 2002b], and transport of solute in rivers [Boano et al., 2007]. CTRW has also been used to understand the observations made in a heterogeneous alluvial aquifer at the Macrodispersion Experiment (MADE) site of the Columbus Air Force Base (Mississippi) [Berkowitz and Scher, 1998] and in a fractured till located on the island of Funen (Denmark) [Kosakowski et al., 2001]. Finally, CTRW was also used for breakthrough curve analysis of numerical experiments mimicking transport in fault zones [O'Brien et al., 2003a,b].

\subsection{Other applications}

As explained in Section 2.4, CTRW has been largely used as average transport model; in fact the ensemble particle motions in many quenched disordered systems obey a CTRW dynamic Berkowitz et al. [2006]. For this reason, motivated by the results of single particle tracking for diffusion in heterogeneous media, CTRW models has been used to study the question of ergodicity in complex media Bel and Barkai [2005], He et al. [2008], Barkai et al. [2012], Metzler et al. [2014]. More recently Dentz et al. [2016b] used TDRW to study the self-averaging properties and ergodicity of subdiffusion in random media. As seen in Section 2.3, TDRW is equivalent to the discretized $\mathrm{ADE}$ and allows to distinguish between ensemble particle motion (CTRW) and diffusive random motion in single realization.

For the extension of RW or TDRW methods to simulate reactivity (possibly in section 3 by extending it to reactivity). Todays, the limits of RW and TDRW methods are found in the reconstruction of concentration fields and their use for reactive transport simulations involving non-linear chemical reactions. Some methods have been developed to reconstruct the concentration field a posteriori from the random walkers while keeping their independence [Fernandez-Garcia and Sanchez-Vila, 2011]. Others decouple the advective and dispersive terms, simulate the advective term with a particle tracking method and the dispersive term with Smoothed Particle Hydrodynamic (SPH) methods Herrera [2009, 2010], Herrera and Beckie [2013]. Each particle is considered as an elementary volume of water containing solutes and exchanging mass with the neighboring volumes according to a prescribed kernel interpolation scheme, which is also eventually used to reconstruct the concentration field. With this method, random walkers remain independent and dispersive processes stem from the particle positions without feedback of the concentration field on the particle displacements. Heterogeneous distributions of particles with rarefaction in low flow zones may critically reduce the accuracy of calculated concentrations. Increasing the particle number or performing particle 
re-meshing are sometimes necessary and impact performances issues or numerical diffusion. To summarize, these methods are intermediary between RW, full SPH, and Particle Strength Exchange (PSE) methods that introduce the influence of concentration fields on the motion of particles and subsequent interdependence of the particles Beaudoin [2002, 2003], Monaghan [2005], Tartakovsky and Meakin [2006]. Like SPH and PSE, RW and TDRW are mesh-free methods that can easily adapt their resolution to local physical or chemical properties without extensive remeshing efforts. When chemistry mostly consists of fluid-rock interactions rather of reactions in solution, particles can again be taken as water volumes or "finite cells" with their embedded solutes chemically interacting with the solid Besnard [2011], Sun [1999, 2002]. It is especially well suited when dispersion is dominated by fluid-rock interactions or mobile-immobile types of exchanges Cirpka [2005], Michalak and Kitanidis [2000]. More generally, appropriate combination of these Lagrangian-based methods could be well suited to locally separate solute dispersion from solute mixing. Dispersion would be simulated as particle movements and mixing as exchanges between particles. The sharing between dispersion and mixing could be derived from some local properties of the particle position or of the reconstructed concentration pattern de Dreuzy [2012], Le Borgne [2011], Borgne and T. [2015]. In this framework, RW and TDRW methods have a strong capacity to integrate detailed physical processes beyond the resolution of the mesh-cell scale.

Finally, RW methods have been used at the small scale to model heat transfer [Emmanuel and Berkowitz, 2007] and emulsion transport in porous media [Cortis and Ghezzehei, 2007]. At a larger scale, additional applications are related to the interpretation of hydraulic pumping tests in heterogeneous porous media [Cortis and Knudby, 2006], to the impact of matrix heterogeneity on the residence times of solutes in fractured media [Robinet et al., 2007], and to the interpretation of water age data sampled under pumping conditions [Leray et al., 2014].

\section{Summary and Conclusions}

At present, RW methods are becoming a mature tool allowing to handle efficiently diffusion processes or advection dispersion in porous media having multiscale quenched heterogeneities, including complex fracture patterns. The scale of interest ranges from molecular and microns using $\mathrm{X} \mu$ scan images of pore space to $\mathrm{km}$ 's characterizing field scales. Radially convergent flows can also be accounted for. These easy to implement class of methods and natural adaptation for HPC make them a powerful tool for up scaling applications. CTRW and TDRW have acquired a rigorous probabilistic connection with finite volume methods. It provides a natural framework for elaborating new theories of normal or anomalous large scale dispersion as it gives a natural picture of mixing and spreading. It highlights the competition between advection, dispersion and the underlying quenched disorder of the medium. The effect of retention and of chemical reactions can be modeled. It yields also practical tools that can be used to carried out within real field studies in order to calibrate some large scale parameters that are needed by industrial simulators.

Future research may take many directions. Finding some robust parameterizations of scattering kernels $M(\mathbf{a}, t ; \mathbf{x}-\mathbf{a})$ or of transfer functions $f($.$) that may$ 
be related efficiently to the medium's disorder could be interesting for applications, [Noetinger and Gautier, 1998, Liu et al., 2000]. Adsorption processes, as well as chemical reactions could also be simulated at least in the linear regime by adding a suitable state variable characterizing the state of the particle during its RW. For example one can consider a two state vector by $\mathbf{x}(t) \rightarrow \mathbf{x}(t)\left[\begin{array}{l}c_{1}(t) \\ c_{2}(t)\end{array}\right]$, in which $c_{1}(t)=0$ or $1,\left(c_{2}(t)=1-c_{1}(t)\right)$ is equal to 1 if the particle is on the first state, 0 else $\left(c_{2}(t)=1-c_{1}(t)\right)$. Choosing suitable transition probabilities for the elementary time step in the corresponding Langevin formulation (so allowing the diffusing particle to change state randomly) provide a direct RW interpretation of the double porosity equations (61). Is it possible to generalize this kind of idea for modeling more complex adsorption or chemical reactions is a possible approach. A big challenge is to be able to generalize RW methods to account for an explicit coupling between the particle's concentration and fluid-flow, leading to viscous fingering patterns. The difficulty is that at a given time the flow depends on the whole particle's concentration map, resulting in a fully coupled flow-transport problem [Saffman and Taylor, 1958, Dagan et al., 1989]. So at first sight, the picture of independent particles undergoing RW seems to be non relevant. Meanwhile, the successful RW picture of Diffusion Limited Aggregation (DLA) [Witten and Sander, 1983, Tang, 1985] corresponding to infinite mobility contrasts fingering gives some hopes that RW will have several applications even in that kind of issues. In particular it could help setting up macroscopic descriptions of the motion of unstable interfaces in a random quenched disorder can be helped using a RW approach using the degrees of freedom describing the front [Nœtinger et al., 2004, Tallakstad et al., 2009, Teodorovich et al., 2011].

\section{A Langevin equation and Fokker Planck equation}

In this section, show the equivalence between the Fokker-Planck equation (1) and the Langevin equation (2). To this end, we use a duality argument. Let $f(\mathbf{x})$ be a twice differentiable function. We consider now the average $\langle f[\mathbf{x}(t)]\rangle$. By virtue of (3), this average may be written as

$$
\langle f[\mathbf{x}(t)]\rangle=\int d \mathbf{x} P(\mathbf{x}, t) f(\mathbf{x})
$$

We consider now $\langle f[\mathbf{x}(t+d t)]\rangle$. To this end, we note that $\mathbf{x}(t+d t)$ is according to (2)

$$
\mathbf{x}(t+d t)=\mathbf{x}(t)+\mathbf{v}[\mathbf{x}(t)] d t+\sqrt{2 \mathbf{B}[\mathbf{x}(t)]} \cdot \mathbf{w}(t) \equiv \mathbf{x}(t)+\Delta \mathbf{x}(t)
$$

where we use the Ito interpretation of the stochastic integral [Risken, 1996]; $\xi(t)$ is a Gaussian random variable with 0 mean and unit variance Taylor expansion of $f[\mathbf{x}(t)+\Delta \mathbf{x}(t)]$ consistently up to order $d t$ then gives

$$
f[\mathbf{x}(t+d t)]-f[\mathbf{x}(t)]=\nabla f[\mathbf{x}(t)] \cdot \mathbf{v}[\mathbf{x}(t)] d t+\nabla \otimes \nabla f[\mathbf{x}(t)]: \mathbf{B}[\mathbf{x}(t)] d t .
$$

It is worthwile noting that this equation is also called the Ito formula, or chain rule of stochastic calculus [Risken, 1996]. Taking the average of (60) and using (58) gives after integration by parts the Fokker-Planck equation (1).

\section{B Dual-porosity models}

Fractured porous media are characterized by a high property contrast between fractures and matrix. This leads to introducing a new class of models, starting from the steady state double 
porosity models [Barenblatt and Zheltov, 1960, Warren and Root, 1963] as derived by [Arbogast et al., 1990, Quintard and Whitaker, 1993] that couple matrix and fracture by means of a linear exchange term.

$$
\left\{\begin{aligned}
\phi_{f} V_{f} \frac{\partial P_{f}(\mathbf{x}, t)}{\partial t} & =D_{f} \nabla^{2} P_{f}(\mathbf{x}, t)+Q(\mathbf{x}, t) \\
\phi_{m} V_{m} \frac{\partial P_{m}(\mathbf{x}, t)}{\partial t} & =D_{m} \nabla^{2} P_{m}(\mathbf{x}, t)-Q(\mathbf{x}, t) .
\end{aligned}\right.
$$

Here, $\phi_{f} V_{f}$ and $\phi_{m} V_{m}$ represent respectively the overall proportions of fracture and matrix volumes (weighted by the relevant porosity and compressibility). The operation $*$ represents a convolution operator. The model is closed once the interporosity flux $Q(\mathbf{x}, t)$ is expressed as a function of $P_{f}(\mathbf{x}, t)$ and $P_{m}(\mathbf{x}, t)$. In the steady state case, $Q(\mathbf{x}, t)$ is given by:

$$
Q(\mathbf{x}, t)=\lambda\left(P_{m}(\mathbf{x}, t)-P_{f}(\mathbf{x}, t)\right) .
$$

The transfer coefficient $\lambda$, reciprocal of a time depends mainly on the geometry of the matrix blocks. It is proportional to $D_{m}$. Its determination from the detailed DFN geometry will be discussed in Section 3.5.

More general models using memory functions accounting for more details of the diffusion inside the matrix can be introduced [Odeh, 1965, Daviau, 1986, x. Chen, 1989, Carslaw and Jaeger, de Swaan, 1976, de Swann and Ramierz-Villa, 1993]. These models belongs to the general class of Multiple Rate Mass Transfer Models (MRMT) models or Multiple Interacting Continua (MINC) [Narasimhan and Pruess, 1988, Haggerty and Gorelick, 1995, r. Dreuzy et al., 2013]. These models correspond to quite different formulations of the same physics differ through the formulation of the exchange term. The latter appears as a time convolution expressed by:

$$
\begin{aligned}
Q(\mathbf{x}, t) & =G(t) *\left(\frac{d\left(P_{m}(\mathbf{x}, t)-P_{f}(\mathbf{x}, t)\right)}{d t}\right) \\
& =\int_{0}^{t} G(t-\tau)\left(\frac{d\left(P_{m}(\mathbf{x}, \tau)-P_{f}(\mathbf{x}, \tau)\right)}{d \tau}\right) d \tau .
\end{aligned}
$$

In all cases, the exchange kernel $G(t)$ is scaled by a parameter $\lambda$ which depends only on the geometry of the matrix blocks. It was shown in [Landereau et al., 2001, Babey et al., 2015] that Multiple porosity models, MRTM models and transient models are equivalent and correspond to different formulations of the same idea.

In most cases, the term $D_{m} \nabla^{2} P_{m}(\mathbf{x}, t)$ may be neglected in the double porosity equations (61), so $P_{m}(\mathbf{x}, t)$ may be eliminated from the equations to provide the following generic form:

$$
\left(\phi_{f} V_{f} \delta(t)+\phi_{m} V_{m} f(t)\right) * \frac{\partial P_{f}(\mathbf{x}, t)}{\partial t}=\nabla \cdot\left(D_{f} \nabla P_{f}(\mathbf{x}, t)\right) .
$$

The quantity $f(t)$ is the time dependent exchange function. Introducing he average pressure in the fractures $<\hat{P}_{f}>(t)$ solution of the following initial value problem:

$$
\begin{array}{r}
\phi(\mathbf{x}) \frac{\partial P_{f}(\mathbf{x}, t)}{\partial t}=\nabla \cdot\left(D(\mathbf{x}) \nabla P_{f}(\mathbf{x}, t)\right) \\
\forall \mathbf{x} \in \Omega_{f} P_{f}(\mathbf{x}, t=0)=1 \\
\forall \mathbf{x} \in \Omega_{m} P_{f}(\mathbf{x}, t=0)=0 \\
<P_{f}>(t)=\frac{1}{\left|\Omega_{f}\right|} \int_{\Omega_{f}} d \mathbf{x} P_{f}(\mathbf{x}, t)
\end{array}
$$

It is possible to show the following relation in the Laplace domain:

$$
<P_{f}>(s)=\frac{\phi_{f} V_{f}}{s\left(\phi_{f} V_{f}+\phi_{m} V_{m} f(s)\right)} .
$$

The practical interest of introducing the $f(s)$ function is that it can be shown that the general solution of a double porosity system (61) can be directly related to a solution of a single porosity equation replacing the argument $s$ of the single porosity solution by the argument $s\left(\phi_{f} V_{f}+s \phi_{m} V_{m} f(s)\right)$. The large amount of analytical single porosity solutions that are well known is sufficient for most practical situations. This means that all the double porosity behavior is captured by $f(s)$, which appears to be a renormalized apparent storativity. The initial value problem (68) defining $\left\langle\hat{P}_{f}\right\rangle(t)$ has in turn a simple RW interpretation. The 
quantity $\left\langle P_{f}\right\rangle(t)$ corresponds to the average proportion of particle undergoing RW (with diffusivity $D_{m}$ ) that belongs to the fractures at time $t$ given they was released at a random location in the fractures at time $t=0$. It was shown by Noetinger, Estebenet and Landereau In the steady state case, the function $f(s)$ is given by :

$$
f(s)=\frac{\lambda}{\phi_{m} V_{m} s+\lambda}
$$

It appears that $\lambda$ is a characteristic diffusion time in the matrix. Explicit expressions may be given for $f(s)$ for either a layered medium, or for spherical blocks:

- for the layered case

$$
f(s)=\sqrt{\frac{\lambda}{3 s V_{m}}} \tanh \sqrt{\frac{3 V_{m} s}{\lambda}} .
$$

- for the spherical case

$$
f(s)=\frac{\lambda}{5 s V_{m}}\left(\sqrt{\frac{15 V_{m} s}{\lambda}} \operatorname{cotanh} \sqrt{\frac{15 \mathrm{~V}_{\mathrm{m} s}}{\lambda}}-1\right)
$$

These generic forms, or others can be used for large scale applications, solving (64) using any numerical approach. It remains to be able to evaluate the transfer coefficient $\lambda$ or the full $f(t)$ function. This is the objective of Section 3.5.

\section{The TDRW algorithm}

Once the diffusion equation (42) has been discretized to get (43), we implement the random walk algorithm as McCarthy did [Carthy, 1993a] first. A detailed derivation of the correctness of the algorithm, as well as its generalization accounting for the presence of traps is presented in Dentz et al [Dentz et al., 2012].

As preliminary step, from the transmissivities we can compute transition probabilities $b_{i j}$ by the definition :

$$
B_{i}=\sum_{j \text { neighbor } i} T_{i j}, \text { and } b_{i j}=\frac{T_{i j}}{B_{i}}
$$

The following relation is satisfied :

$$
\sum_{j \text { neighbor } i} b_{i j}=1
$$

Thereafter, the TDRW algorithm can be presented as follows :

1. choose the number $\mathrm{N}$ of independent walks, and a simulation time $t_{\max }$

2. Choose at random one starting node " $\mathrm{i}$ " according to the source term $s(\mathbf{x}, t=0), \mathrm{k}=1$.

3. at step $\mathrm{k}$ of the algorithm, displace the particle from the site "i" to its nearest neighbor site labeled by "j", with a probability equal to $b_{i j}$.

4. Update the time counter with the relation :

$$
t_{k}=t_{k-1}-\frac{\Delta x^{2}}{B_{i}} \operatorname{Ln}(\text { rand }) .
$$

Here, rand is a number picked at random from the uniform distribution over $[0,1]$.

5. If $t_{k} \leq t_{\max }, k=k+1, i=j$ return to 3 .

6. do this loop $\mathrm{N}$ times to simulate the Brownian motions of $\mathrm{N}$ independent particles.

7. End. 
At the end of the simulation, the user obtains $\mathrm{N}$ arrays containing the jump times $t_{k}$ and the associated particles positions $x\left(t_{k}\right)$. These files can be post processed to compute the statistical quantities of interest, as will be shown later. McCarthy [Carthy, 1993a] showed that the probabilities $P_{i}(t)$ of the particle to be at grid block i at time $\mathrm{t}$ obey the discrete equation (43). When the source term $s(\mathbf{x}, t)$ has the Dirac form, the initial position of the particle corresponds to the grid block in which the source is located. The continuous time character comes from the random character of the jump times.

The main practical interest of this algorithm is that at each iteration, the particle does move to one of its connected neighbors and does never stay at the same node, avoiding unnecessary iterations. The jump time distribution is an exponential distribution whose mean value given by $\frac{\Delta x^{2}}{B_{i}}$ corresponds to a typical diffusion time over the considered grid block.

\section{References}

J. A. Acuna and Y. C. Yortsos. Application of fractal geometry to the study of networks of fractures and their pressure transient. Water Res. Res, 31(3):527-540, 1995.

T. Arbogast, J. Douglas, and U. Hornung. Derivation of the double porosity model of single phase flow via homogeneization theory. SIAM J. Math. Anal, 21(4):823-836, 1990.

L. De Arcangelis, J. Koplik, S. Redner, and D. Wilkinson. Hydrodynamic dispersion in network models of porous media. Phys. Rev. Lett, 57(8):986-999, 1986.

R. Aris. On the dispersion of a solute in a fluid flowing through a tube. In Proceedings of the Royal Society of London A: Mathematical, pages 67-77. Physical and Engineering Sciences. The Royal Society p, 1956.

T. Babey, De Dreuzy J.-r., and C. Casenave. Multi-rate mass transfer (mrmt) models for general diffusive porosity structures. Advances in Water Resources, 76:146-156, 2015. doi: 10.1016/j.advwatres.2014.12.006.

G. I. Barenblatt and Yu.P. Zheltov. Fundamental equations of homogeneous liquids in fissured rocks. Dokl. Akad. Nauk SSSR, 132(3):545-548, 1960.

Eli Barkai, Yuval Garini, and Ralf Metzler. Strange kinetics of single molecules in living cells. Physics Today, 65(8):29-35, 2012. doi: http://dx.doi.org/10.1063/PT.3.1677. URL http://scitation.aip.org/content/aip/magazine/physicstoday/article/65/8/10.1063/PT.3.1677.

D. Barker, J. Ifflander, A. Arya, and Y. Zhang. A parallel algorithm for 3d particle tracking and lagrangian trajectory reconstruction. Measurement science and technology, 23:025301, 2012.

J. Barker. A generalized radial flow model for hydraulic tests in fractured rock. Water Resources Research, 24:1796-1804, 1988.

P. Barthelemy, J. Bertolotti, and D. S. Wiersma. A lévy flight for light. Nature, 453:495-498, 2008.

J. Bear. Dynamics of Fluids in Porous Media. Dover Publications, 1973.

A. Beaudoin and J. R. de Dreuzy. Numerical assessment of 3-D macrodispersion in heterogeneous porous media. Water Resources Research, 49(5):2489-2496, 2013.

A. Beaudoin, De Dreuzy, J. R., J. Erhel, and H. Mustapha. Parallel simulations of underground flow in porous and fractured media paper presented at parallel computing: Current and future issues of high-end computing. NIC., 391:398, 2006.

A. Beaudoin, De Dreuzy, J. R., and J. Erhel. An efficient parallel tracker for advection-diffusion simulations in heterogeneous porous media. In Berlin Springer-Verlag and Heidelberg, editors, Europar, pages 28-31. 2007.

A. Beaudoin, J. R. de Dreuzy, and J. Erhel. Numerical Monte Carlo analysis of the influence of pore-scale dispersion on macrodispersion in 2-D heterogeneous porous media. Water Resources Research, 46:12, 2010.

A. et al. Beaudoin. Anisotropic particle method. Comptes Rendus Mecanique, 330(1):51-56, 2002.

A. et al. Beaudoin. Simulation of anisotropic diffusion by means of a diffusion velocity method. Journal of Computational Physics, 186(1):122-135, 2003.

M. Bechtold, J. Vanderborght, O. Ippisch, and H. Vereecken. Efficient random walk particle tracking algorithm for advective-dispersive transport in media with discontinuous dispersion coefficients and water contents. Water Resources Research, 47:10, 2011. 
M. W. Becker and A. M. Shapiro. Interpreting tracer breakthrough tailing from different forced-gradient tracer experiment configurations in fractured bedrock. Water Resour. Res, 39:1024, 2003.

G. Bel and E. Barkai. Weak ergodicity breaking in the continuous-time random walk. Phys. Rev. Lett., 94:240602, Jun 2005. doi: 10.1103/PhysRevLett.94.240602. URL http://link.aps.org/doi/10.1103/PhysRevLett.94.240602.

B. Berkowitz and I. Balberg. Percolation theory and its application to groundwater hydrology. Water Res. Res, 29(4):775-794, 1993.

B. Berkowitz and H. Scher. Theory of anomalous chemical transport in random fracture networks. Physical Review E, 57(5):5858-5869, 1998.

B. Berkowitz and Harvey Scher. Anomalous transport in random fracture networks. Phys. Rev. Lett., 79:4038-4041, 1997.

B. Berkowitz, H. Scher, and S. Silliman. Anomalous transport in laboratory-scale, heterogeneous porous media. Water Resources Research, 36(1):149-158, 2000.

B. Berkowitz, J. Klafter, R. Metzler, and H. Scher. Physical pictures of transport in heterogeneous media: Advection-dispersion, random-walk, and fractional derivative formulations. Water Resour. Res., 38(10):1191, 2002. doi: doi:10.1029/2001WR001030.

B. Berkowitz, A. Cortis, M. Dentz, and H. Scher. Modeling non-Fickian transport in geological formations as a continuous time random walk. Rev. Geophys., 44(2):RG2003, 2006.

B. et al. Berkowitz. Mass-transfer at fracture intersections - an evaluation of mixing models. Water Resources Research, 30(6):1765-1773, 1994.

K. et al. Besnard. A modified lagrangian-volumes method to simulate nonlinearly and kinetically sorbing solute transport in heterogeneous porous media. Journal of Contaminant Hydrology, 120:89-98, 2011.

B. Bijeljic and M. Blunt. Pore-scale modeling and continuous time random walk analysis of dispersion in porous media. Water Resources Research, 42:1, 2006.

B. Bijeljic, A. Muggeridge, and M. Blunt. Pore-scale modeling of longitudinal dispersion. Water Resources Research, 40:11, 2004.

P. Mostaghimi Bijeljic B. and M. Blunt. Insights into non-fickian solute transport in carbonates. Water Resour. Res., 49:27142728, 2013a.

P. Mostaghimi Bijeljic B., A. Raeini and M. Blunt. Predictions of non-fickian solute transport in different classes of porous media using direct simulation on pore-scale images. Phys. Rev. E, 87(1):013011, 2013b.

F. Boano, A. I. Packman, A. Cortis, R. Revelli, and L. Ridolfi. A continuous time random walk approach to the stream transport of solutes. Water Resources Research, 43:10, 2007.

J. Bodin. From analytical solutions of solute transport equations to multidimensional timedomain random walk (tdrw) algorithms. Water Resources Research, 51(3):1860-1871, 2015.

J. Bodin, G. Porel, and F. Delay. Simulation of solute transport in discrete fracture networks using the time domain random walk method. Earth and Planetary Science Letters, 208 (3-4):297-304, 2003.

J. Bodin, G. Porel, F. Delay, F. Ubertosi, S. Bernard, and J. R. De Dreuzy. Simulation and analysis of solute transport in $2 \mathrm{~d}$ fracture/pipe networks: The solfrac program. Journal of Contaminant Hydrology, 89(1):1-28, 2007.

Le Borgne and Dentz T. M., and villermaux, e., the lamellar description of mixing in porous media. Journal of Fluid Mechanics, 770:458-498, 2015.

J. P. Bouchaud and A. Georges. Anomalous diffusion in disordered media: statistical mechanisms, models and physical applications. Phys. Rep., 195(4):127-293, 1990.

F. Brezzi and M. Fortin. Mixed and Hybrid Finite Element Methods. Springer, Berlin, 1991.

M. Bromly and C. Hinz. Non-fickian transport in homogeneous unsaturated repacked sand. Water Resources Research, 40:7, 2004.

C. Bruderer and Y. Bernab. Network modeling of dispersion: transition from taylor dispersion in homogeneous networks to mechanical dispersion in very heterogeneous ones. Water Resources Research, 37:897-908, 2001.

M. C. Cacas, E. Ledoux, G. de Marsily, A. Barbreau, P. Calmels, B. Gaillard, and R. Margritta. Modeling fracture flow with a stochastic discrete network: Calibration and validation. Water Resources Research, 26:491-500, 1990.

J. et al. Carrera. On matrix diffusion: formulations, solution methods and qualitative effects. Hydrogeology Journal, 6:1, 1998.

H. S. Carslaw and J. C. Jaeger. Conduction of heat in solids. Oxford, at the Clarendon Press. 
J. F. Mc Carthy. Effective permeability of sand-shale reservoirs by a random walk method, j. Phys. A : Math. Gen., 23, 1990.

J. F. Mc Carthy. Analytical models of the effective permeability of sand-shale reservoirs. Geophys. J. Int., 105:513-527, 1991.

J. F. Mc Carthy. Reservoir characterization : Efficient random-walk methods for upscaling and image selection. BHP Research-Melbourne Labs, SPE, 25334, 1993a.

J. F. Mc Carthy. Continuous-time random walks on random media. J. Phys. A : Math. Gen., 26:2495-2503, 1993b.

J. Chang and Y. C. Yortsos. Pressure transient analysis of fractal reservoirs. SPE, 5(1):31-38, 1990.

J. Chang, Y. C. Yortsos, and C. Yanis. Pressure transient analysis of fractal reservoirs. SPE Formation Evaluation, 5:01, 1990.

E. Charlaix, E. Guyon, and S. Roux. Permeability of a random array of fractures of widely varying apertures. Transport in Porous Media, 2:31-43, 1987.

G. Chavent and J. E. Roberts. A unified physical presentation of mixed, mixed-hybrid finite elements and standard finite difference approximations for the determination of velocities in waterflow problems. Advances in Water Resources, 14(6):329-348, 1991.

O. A. Cirpka. Effects of sorption on transverse mixing in transient flows. Journal of Contaminant Hydrology, 78(3):207-229, 2005.

C. Cordes and W. Kinzelbach. Continuous groundwater velocity field and path lines in linear, bilinear and trilinear finite elements. Water Resources Research, 28(11):2903-2911, 1992.

C. Cordes and W. Kinzelbach. Comment on "application of the mixed hybrid finite element approximation in a groundwater flow model: Luxury or necessity?". Water Resources Research, 32(6):1905-1911, 1996.

A. Cortis and B. Berkowitz. Anomalous transport in "classical" soil and sand columns. Soil Science Society of America Journal, 68(5):1539-1548, 2004.

A. Cortis and T. A. Ghezzehei. On the transport of emulsions in porous media. Journal of Colloid and Interface Science, 313(1):1-4, 2007.

A. Cortis and C. Knudby. A continuous time random walk approach to transient flow in heterogeneous porous media. Water Resources Research, 42:10, 2006.

V. Cvetkovic and A. Frampton. Solute transport and retention in three-dimensional fracture networks. Water Resources Research, 48:2, 2012.

V. Cvetkovic, S. Painter, N. Outters, and J. O. Selroos. Stochastic simulation of radionuclide migration in discretely fractured rock near the Äspö Hard Rock Laboratory. Water Resources Research, 40:2, 2004.

Gedeon Dagan et al. Flow and transport in porous formations. Springer-Verlag GmbH \& Co. KG., 1989.

P. V. Danckwerts. The definition and measurements of some characteristics of mixtures, appl. Sci. Res., 3:279-296, 1952.

F. Daviau. Interprétation des essais de puits, les méthodes nouvelles. Publications de l'institut francais du pétrole, Paris, technip edition, 1986

P. De Anna, T. Le Borgne, M. Dentz, A.M. Tartakovsky, D. Bolster, and P. Davy. Flow intermittency, dispersion, and correlated continuous time random walks in porous media. Phys. Rev. Lett., 110(18):184502, 2013.

J. R. de Dreuzy, A. Beaudoin, and J. Erhel. Asymptotic dispersion in 2D heterogeneous porous media determined by parallel numerical simulations. Water Resources Research, 43: $10,2007$.

J. R. et al. de Dreuzy. Advective transport in the percolation backbone in two dimensions. Physical Review E, 64, 2001.

J.-r. et al. de Dreuzy. Time evolution of mixing in heterogeneous porous media. Water Resources Research, 48, 2012.

M. et al. de Simoni. A procedure for the solution of multicomponent reactive transport problems. Water Resources Research, 1141, 2005.

A. de Swaan. Analytic solutions for determining naturally fractured reservoir properties by well testing. SPE J, 5(4):117-22, 1976

A. de Swann and M. Ramierz-Villa. Functions of flow from porous rock blocks. Journal of Petroleum Science and Engineering, 9:39-48, 1993.

F. Delay and J. Bodin. Time domain random walk method to simulate transport by advectiondispersion and matrix diffusion in fractured networks. Geophysical Research Letters, 28(21): 4051-4054, 2001. 
F. Delay, G. Porel, and P. Sardini. Modelling diffusion in a heterogeneous rock matrix with a time-domain lagrangian method and an inversion procedure. Comptes Rendus Geoscience, 334(13):967-973, 2002.

F. Delay, P. Ackerer, and C. Danquigny. Simulating solute transport in porous or fractured formations using random walk particle tracking. Vadose Zone Journal, 4(2):360-379, 2005.

M. Delorme, J. M. Daniel, C. Kada-Kloucha, N. Khvoenkova, S. Schueller, and C. Souque. An efficient model to simulate reservoir stimulation and induced microseismic events on 3D discrete fracture network for unconventional reservoirs. pages 1433-1442. Unconventional Resources Technology Conference (U. R. T. E. C. ), 2013a.

M. Delorme, R. O. Mota, N. Khvoenkova, A. Fourno, and B. Noetinger. A methodology to characterize fractured reservoirs constrained by statistical geological analysis and production: a real field case study. Geological Society, London, Special Publications, 374:374-14, $2013 \mathrm{~b}$.

M. Dentz, A. Cortis, H. Scher, and B. Berkowitz. Time behavior of solute transport in heterogeneous media: transition from anomalous to normal transport. Advances in Water Resources, 27(2):155-173, 2004.

M. Dentz, P. Gouze, A. Russian, J. Dweik, and F. Delay. Diffusion and trapping in heterogeneous media: An inhomogeneous continuous time random walk approach. Advances in Water Resources, 49, 2012.

M. Dentz, A. Russian, and P. Gouze. Self-averaging and ergodicity of subdiffusion in quenched random media. Phys. Rev. E, in press, 2016a.

M. Dentz, A. Russian, and P. Gouze. Self-averaging and ergodicity of subdiffusion in quenched random media. Phys. Rev. E, 2016b.

M. et al. Dentz. Mixing, spreading and reaction in heterogeneous media: a brief review. Journal of Contaminant Hydrology, 120:1-17, 2011.

W. Dershowitz and I. Miller. Dual porosity fracture flow and transport. Geophysical Research Letters, 22(11):1441-1444, 1995.

Y. Edery, A. Guadagnini, H. Scher, and B. Berkowitz. Origins of anomalous transport in heterogeneous media: Structural and dynamic controls. Water Resour. Res., 50: doi:10.1002/2013WR015111, 2014. doi: doi:10.1002/2013WR015111.

A. Einstein. Investigations on the theory of the Brownian movement. Dover Publication, New York, 1956.

S. Emmanuel and B. Berkowitz. Continuous time random walks and heat transfer in porous media. Transport in Porous Media, 67(3):413-430, 2007.

M. Fleury, D. Bauer, and M. C. Néel. Modeling of super-dispersion in unsaturated porous media using nmr propagators. Physical Review E, 87:4-1, 2013. doi: 10.1103/PhysRevE.87.043007.

F. Gjetvaj, A. Russian, P. Gouze, and M. Dentz. Dual control of flow field heterogeneity and immobile porosity on non-fickian transport in berea sandstone. Water Resour. Res., 51, 2015. doi: 10.1002/2015WR017645.

P. Gouze and L. Luquot. X-ray microtomography characterization of porosity, permeability and reactive surface changes during dissolution. J. of Contaminant Hydrol., 120-121:45-55, 2011.

Philippe Gouze, Tanguy Le Borgne, R Leprovost, G Lods, T Poidras, and P Pezard. Nonfickian dispersion in porous media: 1. multiscale measurements using single-well injection withdrawal tracer tests. Water Resources Research, 44(6), 2008a.

Philippe Gouze, Yasmin Melean, Tanguy Le Borgne, Marco Dentz, and Jesus Carrera. Nonfickian dispersion in porous media explained by heterogeneous microscale matrix diffusion. Water Resources Research, 44(11), 2008b.

V. Guillon, M. Fleury, D. Bauer, and M. C. Néel. Superdispersion in homogeneous unsaturated porous media using nmr propagators. Phys. Rev. E, 87(87):4-1, 2013. doi: 10.1103/PhysRevE.87.043007.

V. Guillon, D. Bauer, M. Fleury, and M. C. Néel. Computing the longtime behaviour of nmr propagators in porous media using a pore network random walk model transport in porous media. 10(1007):11242-013, 2014.

R. Haggerty and S. M. Gorelick. Multiple-rate mass transfer for modeling diffusion and surface reactions in media with pore-scale heterogeneity. Water Resour. Res, 31(10):2383-2400, 1995.

R. Haggerty, S. A. McKenna, and L. C. Meigs. On the late time behavior of tracer test breakthrough curves. Water Resour. Res, 36:3467-3479, 2000. 
Y. Hatano and N. Hatano. Dispersive transport of ions in column experiments: An explanation of long-tailed profiles. Water Resources Research, 34(5):1027-1033, 1998.

Y. He, S. Burov, R. Metzler, and E. Barkai. Random time-scale invariant diffusion and transport coefficients. Phys. Rev. Lett., 101:058101, Jul 2008. doi: 10.1103/PhysRevLett.101.058101. URL http://link.aps.org/doi/10.1103/PhysRevLett.101.058101.

P. A. Herrera and R. D. Beckie. An assessment of particle methods for approximating anisotropic dispersion. International Journal for Numerical Methods in Fluids, 71(5):634651, 2013.

P. A. et al. Herrera. A meshless method to simulate solute transport in heterogeneous porous media. Advances in Water Resources, 32(3):413-429, 2009.

P. A. et al. Herrera. A multidimensional streamline-based method to simulate reactive solute transport in heterogeneous porous media. Advances in Water Resources, 33(7):711-727, 2010 .

M. Holzner, V. L. Morales, M. Willmann, and M. Dentz. Intermittent lagrangian velocities and accelerations in three-dimensional porous medium flow. Phys. Rev. E, 92:013015, 2015.

H. Hoteit, J. Erhel, R. Mos, B. Philippe, and P. Ackerer. Numerical reliability for mixed methods applied to flow problems in porous media. Computational Geosciences, 6(2):161194, 2002a.

H. Hoteit, R. Mose, A. Younes, F. Lehmann, and P. Ackerer. Three-dimensional modeling of mass transfer in porous media using the mixed hybrid finite elements and the random-walk methods. Mathematical Geology, 34(4):435-456, 2002b.

F. Jimenez-Hornero, J. Giraldez, A. Laguna, and Y. Pachepsky. Continuous time random walks for analyzing the transport of a passive tracer in a single fissure. Water Resources Research, 41:4, 2005.

B. Kaludercic. Parallelisation of the lagrangian model in a mixed eulerian-lagrangian cfd algorithm. Journal of Parallel and Distributed Computing, 64(2):277-284, 2004.

P. K. Kang, M. Dentz, T. Le Borgne, and R. Juanes. Spatial Markov model of anomalous transport through random lattice networks. Phys. Rev. Lett., 107:180602, doi:10.1103/PhysRevLett.107.180602, 2011.

P. K. Kang, P. de Anna, J. P. Nunes, B. Bijeljic, M. Blunt, and R. Juanes. Pore-scale intermittent velocity structure underpinning anomalous transport through $3 \mathrm{~d}$ porous media. Geophys. Res. Lett., 41:doi:10.1002/2014GL061475, 2014.

P. K. Kang, T. Le Borgne, T. Dentz, O. Bour, and R. Juanes. Impact of velocity correlation and distribution on transport in fractured media: field evidence and theoretical model. Water Resour. Res, 51:940-959, 2015.

V. M. Kenkre, E. W. Montroll, and M. F. Shlesinger. Generalized master equations for continuous-time random walks. J. Stat. Phys., 9(1):45-50, 1973.

N. Khvoenkova and M. Delorme. An optimal method to model transient flows in 3d discrete fracture network. IAMG conference, 2011:1238-1249, 2011.

I. C. Kim and S. Torquato. Effective conductivity of suspensions of overlapping spheres. Journal of applied physics, 71(6):2727-2735, 1992.

W. Kinzelbach. The random walk method in pollutant transport simulation. In Groundwater flow and quality modelling, pages 227-245. Springer, 1988.

W. Kinzelbach and G. Uffink. The random walk method and extensions in groundwater modelling, volume Transport Processes in Porous Media. Springer, Netherlands, 1991.

P. Kitanidis. The concept of the dilution index. Water Resources Research, 34:1181-93, 1994.

J. Klafter and R. Silbey. Derivation of the continuous-time random-walk equation. Phys. Rev. Lett., 44:55-58, 1980.

J. Klafter and I. Sokolov. Anomalous diffusion spreads its wings. Phys. World, 18(8):29-32, 2005.

J. Koplik, S. Redner, and D. Wilkinson. Transport and dispersion in random networks with percolation disorder. Physical Review A, 37(7):2619-2636, 1988.

G. Kosakowski. Anomalous transport of colloids and solutes in a shear zone. Journal of Contaminant Hydrology, 72(1-4):23-46, 2004.

G. Kosakowski and B. Berkowitz. Flow pattern variability in natural fracture intersections. Geophysical Research Letters, 26:12, 1999.

G. Kosakowski, B. Berkowitz, and H. Scher. Analysis of field observations of tracer transport in a fractured till. Journal of Contaminant Hydrology, 47(1):29-51, 2001.

P. Landereau, B. Noetinger, and M. Quintard. Quasi-steady two-equation models for diffusive transport in fractured porous media: large-scale properties for densely fractured systems. 
Adv. in Water Resour., 24(8):863-876, 2001.

et al. Le Borgne, T. Persistence of incomplete mixing: A key to anomalous transport. Physical Review E, 84(1):015301, 2011.

T. Le Borgne and P. Gouze. Non-fickian dispersion in porous media: 2. model validation from measurements at different scales. Water Resour. Res, 44:W06427, 2008.

T. Le Borgne, M. Dentz, and J. Carrera. Spatial markov processes for modeling lagrangian particle dynamics in heterogeneous porous media. Phys. Rev. E, 78:041110, $2008 \mathrm{a}$.

T. Le Borgne, M. Dentz, and J. Carrera. A Lagrangian statistical model for transport in highly heterogeneous velocity fields. Phys. Rev. Lett., 101:090601, 2008b.

T. Le Borgne, M. Dentz, and E. Villermaux. Stretching, coalescence and mixing in porous media. Phys. Rev. Lett., 110:204501, 2013.

et al. Le Borgne T. Non-fickian mixing: Temporal evolution of the scalar dissipation rate in heterogeneous porous media. Advances in Water Resources, 33:1468-1475, 2010.

S. Leray, J. R. de Dreuzy, L. Aquilina, V. Vergnaud-Ayraud, T. Labasque, O. Bour, and T. Le Borgne. Temporal evolution of age data under transient pumping conditions. Journal of Hydrology, 511:555-566, 2014.

H. Liu, Y. Zhang, Q. Zhou, and F. Molz. An interpretation of potential scale dependence of the effective matrix diffusion coefficient. Journal of Contaminant Hydrology, 90(1):41-57, 2007.

H. H. Liu, G. S. Bodvarsson, and L. Pan. Determination of particle transfer in random walk particle methods for fractured porous media. Water Resources Research, 36(3):707-713, 2000.

G. Matheron and G. De. Marsily is transport in porous media always diffusive? a counterexample. Water Resour. Res, 16(5):901-917, 1980.

R. Metzler and J. J. Klafter. The random walk's guide to anomalous diffusion: a fractional dynamics approach. Physics reports, 339:1, 2000. doi: http://dx.doi.org/10.1016/S03701573(00)00070-3.

R. Metzler, W. G. W. G. Glockle, and T. F. Nonnenmacher. Fractional model equation for anomalous diffusion. Physica A: Statistical Mechanics and its Applications, 211:1, 1994. doi: 10.1016/0378-4371(94)90064-7.

Ralf Metzler, Jae-Hyung Jeon, Andrey G Cherstvy, and Eli Barkai. Anomalous diffusion models and their properties: non-stationarity, non-ergodicity, and ageing at the centenary of single particle tracking. Physical Chemistry Chemical Physics, 16(44):24128-24164, 2014.

A. M. Michalak and P. K. Kitanidis. Macroscopic behavior and random-walk particle tracking of kinetically sorbing solutes. Water Resources Research, 36(8):2133-2146, 2000.

J. J. Monaghan. Smoothed particle hydrodynamics, rep. Prog. Phys., 68, 2005.

R. Mosé, P. Siegel, P. Ackerer, and G. Chavent. Application of the mixed hybrid finite element approximation in a groundwater model: Luxury or necessity? Water Resources Research, 30:3001-3012, 1994.

T. N. Narasimhan and K. Pruess. Minc: An approach for analyzing transport in strongly heterogeneous systems. In Groundwater Flow and Quality Modeling, editors, Springer Netherlands, pages 375-391. 224, 1988.

M. C. Néel, S. H. Rakotonasyl, D. Bauer, M. Joelson, and M. Fleury. All order moments and other functionals of the increments of some non-markovian processes. Journal of Statistical Mechanics: Theory and Experiment, 2011, February 2011.

M. C. Néel, D. Bauer, and M. Fleury. Model to interpret pulsed-field-gradient nmr data including memory and superdispersion effects. Phys. Rev. E, 1(10):62121-06213, 2014.

B. Noetinger and T. Estebenet. Up scaling of fractured porous media using a continuous time random walk method. Transport in Porous Med, 39:315-337, 2000.

B. Noetinger and Y. Gautier. Use of the fourier-laplace transform and of diagrammatical methods to interpret pumping tests in heterogeneous reservoirs. Advances in water resources, 21 (7):581-590, 1998.

B. Noetinger and N. Jarrige. A quasi steady state method for solving transient darcy flow in complex 3d fractured networks. J Comput. Phys, 231(1):23-38, 2012.

B. Noetinger, T. Estebenet, and P. Landereau. A direct determination of the transient exchange term of fractured media using a continuous time random walk method. Transport in Porous Med, 44:539-557, 2001a.

B. Noetinger, T. Estebenet, and M. Quintard. Up scaling of fractured media: Equivalence between the large scale averaging theory and the continuous time random walk method. Transport in Porous Med, 43:581-596, $2001 \mathrm{~b}$. 
Benoît Nœetinger, Vincent Artus, and Ludovic Ricard. Dynamics of the water-oil front for two-phase, immiscible flow in heterogeneous porous media. 2-isotropic media. Transport in porous media, 56(3):305-328, 2004.

J. P. Nunes, B. Bijeljic, and M. J. Blunt. Time-of-flight distributions and breakthrough curves in heterogeneous porous media using a pore-scale streamline tracing algorithm. Transport in Porous Media, 109(2):317-336, 2015.

G. O'Brien, C. Bean, and F. McDermott. A numerical study of passive transport through fault zones. Earth and Planetary Science Letters, 214(3-4):633-643, 2003a.

G. O'Brien, C. Bean, and F. McDermott. Numerical investigations of passive and reactive flow through generic single fractures with heterogeneous permeability. Earth and Planetary Science Letters, 213(3-4):271-284, 2003b.

A. S. Odeh. Unsteady-state behavior of naturally fractured reservoirs. SPE Journal, pages $60-66,1965$

B. O'Shaughnessy and I. I. Procaccia. Diffusion on fractals. Physical Review A, 32:3073-3083, 1985. doi: 10.1103/PhysRevA.32.3073.

J. M. Ottino. The kinematics of mixing: stretching, chaos and transport. Cambridge University Press, 1989.

S. Painter and V. Cvetkovic. Upscaling discrete fracture network simulations: An alternative to continuum transport models. Water Resources Research, 41:2, 2005.

S. Painter, V. Cvetkovic, J. Mancillas, and O. Pensado. Time domain particle tracking methods for simulating transport with retention and first-order transformation. Water Resources Research, 44:1, 2008.

L. Pan and G. S. Bodvarsson. Modeling transport in fractured porous media with the randomwalk particle method: The transient activity range and the particle transfer probability. Water Resources Research, 38:6, 2002a.

L. H. Pan and G. S. Bodvarsson. Modeling transport in fractured porous media with the random-walk particle method: The transient activity range and the particle transfer probability. Water Resources Research, 38(6):1080, 2002b.

Y.-j. et al. Park. Transport and intersection mixing in random fracture networks with power law length distributions. Water Resources Research, 37(10):2493-2501, 2001.

Y.-j. et al. Park. Transport behavior in three-dimensional fracture intersections. Water Resources Research, 39:1, 2003.

G. Pichot, J. Erhel, and J. R. De Dreuzy. A mixed hybrid mortar method for solving flow in discrete fracture networks. Applicable Analysis, 89(10):1729-1643, 2010.

P.P. Mitra P.N. Sen, L.M. Schwartz and B.I. Halperin. Surface relaxation and the long-time diffusion coefficient in porous media: Periodic geometries. Phys. Rev. B, 49(1):215-225, 1994.

D. W. Pollock. Semianalytical computation of path lines for finite-difference models. Ground Water, 26(6):743-750, 1988.

Zheng-Xian Qu, Zhi-Feng Liu, Xiao-Hong Wang, and Peng Zhao. Finite analytic numerical method for solving two-dimensional quasi-laplace equation. Numerical Methods for Partial Differential Equations, 30:6, 2014.

M. Quintard and S. Whitaker. One- and two-equation models for transient diffusion processes in two-phase systems. Advances in Heat Transfer, 23:369-464, 1993.

J r. Dreuzy, A. Rapaport, T. Babey, and J. Harmand. Influence of porosity structures on mixing-induced reactivity at chemical equilibrium in mobile/immobile multi-rate mass transfer (mrmt) and multiple interacting continua (minc) models. Water Resour. Res, 49 (12):8511-8530, 2013.

H. Risken. The Fokker-Planck Equation. Springer Heidelberg New York, 1996.

C. Rivard and F. Delay. Simulations of solute transport in fractured porous media using 2D percolation networks with uncorrelated hydraulic conductivity fields. Hydrogeology Journal, 12(6):613-627, 2004.

J.-e. Roberts and J. m. Thomas. Mixed and hybrid methods. In Handbook of Numerical Analysis 2, Finite Element Methods -part 1, pages 523-639. Elsevier Science Publishers B.V. (North-Holland), 1991.

J. C. Robinet, P. Sardini, F. Delay, and K. H. Hellmuth. The effect of rock matrix heterogeneities near fracture walls on the residence time distribution (RTD) of solutes. Transport in Porous Media, 72(3):393-408, 2007.

R. K. Romeu and B. Noetinger. Calculation of internodal transmissivities in finite difference models of flow in heterogeneous porous media. Water Resources Research, 31(4):943-959, 
1995.

D. Roubinet and J. Irving. Discrete-dual-porosity model for electric current flow in fractured rock. Journal of Geophysical Research-Solid Earth, 119(2):767-786, 2014.

D. Roubinet, H. H. Liu, and J. R. de Dreuzy. A new particle-tracking approach to simulating transport in heterogeneous fractured porous media. Water Resources Research, 46:11, 2010.

D. Roubinet, J. R. de Dreuzy, and D. M. Tartakovsky. Particle-tracking simulations of anomalous transport in hierarchically fractured rocks. Computers \& Geosciences, 50:52-58, 2013.

A. Russian, M. Dentz, and P. Gouze. Time domain random walks for hydrodynamic transport in heterogeneous media. Water Resour. Res., page under review, 2016.

Philip Geoffrey Saffman and Geoffrey Taylor. The penetration of a fluid into a porous medium or hele-shaw cell containing a more viscous liquid. In Proceedings of the Royal Society of London A: Mathematical, Physical and Engineering Sciences, volume 245, pages 312-329. The Royal Society, 1958.

M. Sahimi. Flow and transport in porous media and fractured rock: from classical methods to modern approaches. John Wiley \& Sons, 2011.

P. Salamon, D. Fern ndez Garcia, and J. J. Gmez-Hernndez. A review and numerical assessment of the random walk particle tracking method. Journal of Contaminant Hydrology, 87(3-4): 277-305, 2006.

H. Scher and M. Lax. Stochastic transport in a disordered solid. I. Theory. Phys. Rev. B, 7 (1):4491-4502, 1973a.

H. Scher and M. Lax. Stochastic transport in a disordered solid. ii. Impurity conduction. Phys. Rev. B, 7:4502-4519, 1973b.

H. Scher, G. Margolin, and B. Berkowitz. Towards a unified framework for anomalous transport in heterogeneous media. Chemical Physics, 284:349-359, 2002a.

H. Scher, G. Margolin, R. Metzler, J. Klafter, and B. Berkowitz. The dynamical foundation of fractal stream chemistry: The origin of extremely long retention times. Geophysical Research Letters, 29:5, 2002b.

K. Semra, P. Ackerer, and R. Mosé. Three dimensional groundwater quality modeling in heterogeneous media. In Water pollution II: Modeling, pages 3-11. measuring and prediction, Computational Mechanics Publications, Southampton, UK, 1993.

P.N. Sen. Time-dependent diffusion coefficient as a probe of permeability of the pore-wall. $J$. Chem. Phys., 19:9871, 2003.

P.N. Sen. Time-dependent diffusion coefficient as a probe of geometry. Concepts in Magnetic Resonance Part A, 23A:1-21, 2004.

G. Srinivasan, D. M. Tartakosky, M. Dentz, H. Viswanathan, B. Berkowitz, and B. A. Robinson. Random walk particle tracking simulations of non-fickian transport in heterogeneous media. J. Comp. Phys., 229:4304-4314, 2010.

N.-z. Sun. A finite cell method for simulating the mass transport process in porous media. Water Resources Research, 35(12):3649-3662, 1999.

N. Z. Sun. Modeling biodegradation processes in porous media by the finite cell method. Water Resources Research, 38:3, 2002.

S. Redner Superdiffusive. Transport due to random velocity fields. Physica D, 38:287, 1989.

Ken Tore Tallakstad, Henning Arendt Knudsen, Thomas Ramstad, Grunde Lovoll, Knut Jorgen Maloy, Renaud Toussaint, and Eirik Grude Flekkoy. Steady-state two-phase flow in porous media: statistics and transport properties. Physical review letters, 102(7):74502, 2009.

Chao Tang. Diffusion-limited aggregation and the saffman-taylor problem. Physical Review A, 31(3):1977, 1985.

D. H. Tang, E. O. Frind, and E. A. Sudicky. Contaminant transport in fractured porous media: Analytical solution for a single fracture. Water Resources Research, 17(3):555-564, 1981.

A. M. Tartakovsky and P. Meakin. Pore scale modeling of immiscible and miscible fluid flows using smoothed particle hydrodynamics. Advances in Water Resources, 29(10):1464-1478, 2006.

G. I. Taylor. Diffusion and mass transport in tubes. Proceedings of the Physical Society. Section B, 67:857, 1954.

EV Teodorovich, PE Spesivtsev, and B Nœtinger. A stochastic approach to the two-phase displacement problem in heterogeneous porous media. Transport in Porous Media, 87(1): 151-177, 2011.

A. F. B. Tompson and L. W. Gelhar. Numerical simulation of solute transport in threedimensional, randomly heterogeneous porous media. Water Resources Research, 26(10): 
2541-2562, 1990.

G. J. M. Uffink. A random walk method for the simulation of macrodispersion in a stratified aquifer, paper presented at i. In 18th Gen. Assembly Proc. Symp., IAHS Publ. 146. IAHS, Wallingford, UK., Hamburg, Germany, 1985.

Yan-Feng Wang, Zhi-Feng Liu, and Xiao-Hong Wang. Finite analytic numerical method for three-dimensional fluid flow in heterogeneous porous media. Journal of Computational Physics, 278:169, 2014.

J. E. Warren and P. J. Root. The behavior of naturally fractured reservoirs. The Society of Petroleum Engineers Journal, 3:245-255, 1963.

X. H. Wen and J. J. Gomez-Hernandez. The constant displacement scheme for tracking particles in heterogeneous aquifers. Ground Water, 34(1):135-142, 1996.

M. et al. Willmann. Transport upscaling in heterogeneous aquifers: What physical parameters control memory functions? Water Resources Research, 44:12, 2008.

Thomas A Witten and Leonard M Sander. Diffusion-limited aggregation. Physical Review B, $27(9): 5686,1983$.

Z. x. Chen. Transient flow of slightly compressible fluids through double-porosity, doublepermeability systems - a state-of-the-art review. Transport in Porous Media, 4:147-184, 1989.

C. Zheng and G. D. Bennett. Applied contaminant transport modeling. $440 \mathrm{pp}$, Wiley Interscience, 2nd edition, 2002.

S. Zimmermann, P. Koumoutsakos, and W. Kinzelbach. Simulation of pollutant transport using a particle method. Journal of Computational Physics, 173(1):322-347, 2001. 